\title{
Radiative neutrino masses in the $\nu_{R}$ MSSM
}

\author{
Pablo Candia da Silva $\odot^{*}$ and Apostolos Pilaftsis $\odot^{\dagger}$ \\ Consortium for Fundamental Physics, School of Physics and Astronomy, University of Manchester, \\ Manchester M13 9PL, United Kingdom
}

(Received 4 September 2020; accepted 8 October 2020; published 12 November 2020)

\begin{abstract}
We present a complete analysis of scenarios with radiatively generated neutrino masses that may occur in the minimal supersymmetric Standard Model with low-scale right-handed neutrinos. For brevity, we call such a model the $\nu_{R}$ MSSM. We pay particular attention to the impact of the nonrenormalization theorem of supersymmetry (SUSY) on the loop-induced neutrino masses, by performing our computations in the weak and flavor bases. In particular, we find that the smallness of the observed light neutrino masses may naturally arise due to a soft SUSY-screening effect from a nearly supersymmetric singlet neutrino sector. The profound phenomenological and cosmological implications that may originate from this screening phenomenon in the $\nu_{R}$ MSSM and its minimal extensions are discussed.
\end{abstract}

DOI: 10.1103/PhysRevD.102.095013

\section{INTRODUCTION}

Supersymmetry (SUSY) [1,2] is an elegant theoretical framework which aspires to technically address several problems that are central in particle physics and cosmology, such as the infamous gauge-hierarchy problem, the unification of gauge couplings and the nature of the dark matter (DM) in the Universe [3]. In its exact realization, SUSY is endowed with powerful nonrenormalization theorems that forbid the presence of new operators in the so-called superpotential to all orders in loop expansion [4]. Even beyond the tree level, the existing superpotential operators do not require renormalization other than the one that arises from the wave functions of the fields involved [5]. However, for phenomenological reasons, SUSY needs to be broken at scales larger than the electroweak (EW) scale, at least higher than TeV [6]. If this breaking is "soft" through holomorphic operators of energy dimensions 3 and less, then this softly broken SUSY will still help to eliminate all quadratically sensitive ultraviolet (UV) divergences in the superpotential. This property is crucial in stabilizing the EW scale against quantum corrections of new physics (other than gravity) that may take place at much higher scale, e.g., at scales of gauge-coupling unification in grand unified theories (GUTs) [7] whose low-energy limit includes the Standard Model (SM).

\footnotetext{
*pablo.candiadasilva@postgrad.manchester.ac.uk apostolos.pilaftsis@manchester.ac.uk
}

Published by the American Physical Society under the terms of the Creative Commons Attribution 4.0 International license. Further distribution of this work must maintain attribution to the author(s) and the published article's title, journal citation, and DOI. Funded by SCOAP ${ }^{3}$.
A minimal realization of softly broken SUSY is the socalled minimal supersymmetric Standard Model (MSSM) [8]. As a remnant of the nonrenormalization SUSY theorems mentioned above, there exist now regions of parameter space for which physical observables vanish or become very suppressed, while they are forbidden in the exact SUSY limit. Typical examples in which this soft SUSY-screening phenomenon occurs are lepton- and quark-flavor-violating decay processes, such as $b \rightarrow s \gamma$ [9] and $\mu \rightarrow e \gamma$ [10-12], as well as flavor-conserving observables, such as electric and anomalous magnetic dipole moments of leptons $[13,14]$.

In this paper we show that a similar soft SUSY-screening phenomenon may be the origin of the smallness of the observed light neutrino masses [15-17] in theories, in which light neutrino masses are forbidden at the tree level. To explicitly demonstrate this screening phenomenon, we study typical scenarios that may occur in the MSSM with a number $n_{R}$ of electroweak- or TeV-scale right-handed (singlet) neutrinos $\nu_{i R}$ (with $i=1,2, \ldots, n_{R}$ ). For brevity, we call such a model the $\nu_{R}$ MSSM. To be able to have good control of the SUSY-screening effect on the loop-induced neutrino masses, we perform our computations of the contributing Feynman graphs in the weak and flavor bases, rather than in the mass basis.

A widely explored framework accounting for the origin of the very small neutrino masses is given by the so-called seesaw mechanism [18-26]. This mechanism relies upon the hypothesis that neutrinos are Majorana fermions [27]. In its most popular implementation, the Type-I seesaw scenario [18-23] postulates the existence of right-handed neutrinos, $\nu_{i R}$, which are singlets under the SM gauge group. This scenario leads to an effective neutrino mass matrix $\mathbf{m}_{\nu}$, which is parametrically suppressed by the 
lepton-number-violating (LNV) mass matrix of the singlet neutrinos $\mathbf{m}_{M}$, i.e.,

$$
\mathbf{m}_{\nu}=-\mathbf{m}_{D} \mathbf{m}_{M}^{-1} \mathbf{m}_{D}^{\top}
$$

where $\mathbf{m}_{D}$ is the Dirac mass matrix which is generated after spontaneous symmetry breaking (SSB) by the vacuum expectation value (VEV) of the SM Higgs doublet $v_{\mathrm{SM}} \approx 246 \mathrm{GeV}$. For $\mathbf{m}_{D} \sim v_{\mathrm{SM}}$, the seesaw formula (1.1) implies that $\mathbf{m}_{M}$ should be of order $10^{14} \mathrm{GeV}$, namely close to the GUT scale, in order to account for the observed sub$\mathrm{eV}$ neutrino masses. However, a phenomenological difficulty of such a scenario is that the required high-scale of the seesaw mechanism renders its LNV singlet sector not directly testable in any foreseeable experiment.

The above difficulty may be circumvented in low-scale seesaw models [28], where the LNV scale as dictated by the size of the mass matrix $\mathbf{m}_{M}$ is closer to the EW scale. Typical examples are the inverse seesaw scenario (ISS) $[29,30]$, or radiative seesaw scenarios where the neutrino masses are absent at the tree level, but they are generated at loop level [31-34]. For a comprehensive review, the interested reader may consult [35]. As mentioned above, SUSY-screening is another mechanism to naturally predict light neutrino masses, without introducing an unnecessary disparity between the LNV and EW scales, thereby giving rise to a LNV sector that could be directly probed at highenergy colliders [28,36-42]. Here, we explicitly demonstrate this phenomenon within the context of a few representative scenarios in the $\nu_{R}$ MSSM. Unlike previous studies [43-45], we calculate the complete set of diagrammatic contributions that come from both the ordinary SM sector [31] (called here the $\nu_{R} \mathrm{SM}$ ) and its SUSY counterpart. In so doing, we pay particular attention to the impact of the SUSY nonrenormalization theorem on the loopinduced neutrino masses.

The paper is organized as follows. After this introductory section, we describe the $\nu_{R}$ MSSM in Sec. II, including our conventions and notations for the field content. In Sec. III, we calculate the one-loop induced neutrino masses, by performing our computations in the weak and flavor bases. In particular, we check the vanishing of the neutrino masses in the exact SUSY limit, independently of whether LNV mass parameters are present in the superpotential or not. In this way, we can identify the regions of parameter space for soft SUSY screening. The latter guides our analysis in Sec. IV, where we show numerical results for particular radiative neutrino scenarios in the $\nu_{R}$ MSSM. Section V summarizes our conclusions, as well as presents future research directions. All technical details pertinent to the $\nu_{R}$ MSSM and our calculations are given in Appendices A-C.

\section{THE $\nu_{R}$ MINIMAL SUPERSYMMETRIC STANDARD MODEL}

As mentioned in the Introduction, the $\nu_{R}$ MSSM is obtained by adding a number of $n_{R}$ left-chiral superfields $\hat{N}_{i}^{C}$ (with $i=1,2, \ldots, n_{R}$ ) to the field content of the MSSM. There are several studies of the $\nu_{R}$ MSSM in the literature [43,46-53]. In our paper, we adhere to the notation displayed in Table I for the full $\nu_{R}$ MSSM spectrum of fields.

The superpotential of the $\nu_{R}$ MSSM is given by

$$
W=W_{\mathrm{MSSM}}+\hat{L} i \sigma_{2} \hat{H}_{u} \mathbf{Y}_{\nu} \hat{N}^{C}+\frac{1}{2} \hat{N}^{C} \mathbf{m}_{M} \hat{N}^{C},
$$

where $W_{\text {MSSM }}$ is the usual MSSM superpotential, $\mathbf{Y}_{\nu}$ and $\mathbf{m}_{M}$ denote the $3 \times n_{R}$ neutrino Yukawa matrix and the

TABLE I. Particle content of the $\nu_{R}$ MSSM. The numbers in boldface indicate the dimension of the gauge group representation under which each multiplet transforms. Here, $\sigma_{i}$ with $i=1,2,3$ are the usual Pauli matrices, while $\lambda^{a}$ with $a=1,2, \ldots, 8$ are the Gell-Mann matrices.

\begin{tabular}{lccc}
\hline \hline Superfields & Bosons & Fermions & $\mathrm{SU}(3)_{c} \otimes \mathrm{SU}(2)_{L} \otimes U(1)_{Y}$ \\
\hline Gauge multiplets & $G_{\mu}^{a} \frac{1}{2} \lambda^{a}$ & $(\mathbf{8}, \mathbf{1}, 0)$ \\
$\hat{G}^{a}$ & $W_{\mu}^{i} \frac{1}{2} \sigma_{i}$ & $\tilde{g}^{a}$ & $(\mathbf{1}, \mathbf{3}, 0)$ \\
$\hat{W}^{a}$ & $B_{\mu}$ & $\tilde{W}^{i}$ & $(\mathbf{1}, \mathbf{1}, 0)$ \\
$\hat{B}$ & & $\tilde{B}_{0}$ & \\
Matter multiplets & $\tilde{L}^{\top}=\left(\tilde{\nu}_{L}, \tilde{e}_{L}\right)$ & $L^{\top}=\left(\nu_{L}, e_{L}\right)$ & $(\mathbf{1}, \mathbf{2},-1)$ \\
$\hat{L}$ & $\tilde{e}_{R}^{*}$ & $e_{R}^{C}$ & $(\mathbf{1}, \mathbf{1}, 2)$ \\
$\hat{E}^{C}$ & $\tilde{Q}^{\top}=\left(\tilde{u}_{L}, \tilde{d}_{L}\right)$ & $Q^{\top}=\left(u_{L}, d_{L}\right)$ & $(\mathbf{3}, \mathbf{2}, 1 / 3)$ \\
$\hat{Q}$ & $\tilde{u}_{R}^{*}$ & $u_{R}^{C}$ & $(\mathbf{3}, \mathbf{1},-4 / 3)$ \\
$\hat{U}^{C}$ & $\tilde{d}_{R}^{*}$ & $d_{R}^{C}$ & $(\mathbf{3}, \mathbf{1}, 2 / 3)$ \\
$\hat{D}^{C}$ & $H_{d}^{\top}=\left(H_{d}^{0}, H_{d}^{-}\right)$ & $\tilde{h}_{d}^{\top}=\left(\tilde{h}_{d}^{0}, \tilde{h}_{d}^{-}\right)$ & $(\mathbf{1}, \mathbf{2},-1)$ \\
$\hat{H}_{d}$ & $H_{u}^{\top}=\left(H_{u}^{+}, H_{u}^{0}\right)$ & $\tilde{h}_{u}^{\top}=\left(\tilde{h}_{u}^{+}, \tilde{h}_{u}^{0}\right)$ & $(\mathbf{1}, \mathbf{2}, 1)$ \\
$\hat{H}_{u}$ & $\tilde{\nu}_{R}^{*}$ & $\nu_{R}^{C}$ & $(\mathbf{1}, \mathbf{1}, 0)$ \\
$\hat{N}^{C}$ & & &
\end{tabular}


$n_{R} \times n_{R}$ Majorana mass matrix, respectively. These latter matrices also appear in the $\nu_{R} \mathrm{SM}$. Note that we use boldface format to highlight matrices with flavor structure. Employing superspace techniques [54], we can derive the SUSY Yukawa Lagrangian of interest to us,

$$
\begin{aligned}
-\mathcal{L}_{\mathrm{Y}}= & \bar{L}^{C} i \sigma_{2} H_{u} \mathbf{Y}_{\nu} \nu_{R}^{C}+\tilde{L}^{\top} i \sigma_{2} \tilde{h}_{u} \mathbf{Y}_{\nu} \nu_{R}^{C}+\bar{L}^{C} i \sigma_{2} \tilde{h}_{u} \mathbf{Y}_{\nu} \tilde{\nu}_{R}^{*} \\
& +\frac{1}{2} \bar{\nu}_{R} \mathbf{m}_{M} \nu_{R}^{C}+\text { H.c. }
\end{aligned}
$$

Likewise, from the superpotential (2.1), one may derive the $F$-term contributions from the right-handed sneutrinos $\tilde{\nu}_{R}$ to the scalar potential,

$$
\begin{aligned}
V_{F}^{\tilde{\tau}_{R}}= & \tilde{\nu}_{R}^{*} \mathbf{Y}_{\nu}^{\top} \mathbf{Y}_{\nu}^{*} \tilde{\nu}_{R} H_{u}^{\dagger} H_{u}-\left(\tilde{e}_{R}^{*} \mathbf{Y}_{e}^{\top} \mathbf{Y}_{\nu}^{*} \tilde{\nu}_{R} H_{u}^{\dagger} H_{d}+\text { H.c. }\right) \\
& +\tilde{L}^{\top} \mathbf{Y}_{\nu} \tilde{\nu}_{R}^{*} \tilde{\nu}_{R} \mathbf{Y}_{\nu}^{\dagger} \tilde{L}^{*}-\left(\mu \tilde{\nu}_{R} \mathbf{Y}_{\nu}^{\dagger} \tilde{L}^{\dagger} H_{d}+\text { H.c. }\right) \\
& -H_{u}^{\top} i \sigma_{2} \tilde{L} \mathbf{Y}_{\nu} \mathbf{Y}_{\nu}^{\dagger} \tilde{L}^{\dagger} i \sigma_{2} H_{u}^{*}+\left(\tilde{L}^{\top} i \sigma_{2} H_{u} \mathbf{Y}_{\nu} \mathbf{m}_{M}^{\dagger} \tilde{\nu}_{R}+\text { H.c. }\right) \\
& +\tilde{\nu}_{R}^{*} \mathbf{m}_{M} \mathbf{m}_{M}^{\dagger} \tilde{\nu}_{R} .
\end{aligned}
$$

Observe that the $F$-term induced potential $V_{F}^{\tilde{\nu}_{R}}$ contains the LNV operator, $\tilde{L}^{\top} i \sigma_{2} H_{u} \widetilde{\nu}_{R}$ given by the penultimate term on the right-hand side (rhs) of (2.3), which violates the lepton number by two units. As we will discuss in the next section, this SUSY-generated LNV operator plays an instrumental role in the determination of the radiative neutrino masses by screening the effect of the ordinary right-handed neutrinos from the $\nu_{R} \mathrm{SM}$.

Finally, the relevant soft SUSY-breaking Lagrangian derivable from the superpotential (2.1) is given by

$$
\begin{aligned}
-\mathcal{L}_{\text {soft }}= & -\mathcal{L}_{\text {soft }}^{\mathrm{MSSM}}+\tilde{\nu}_{R}^{*} \mathbf{m}_{\tilde{\nu}}^{2} \tilde{\nu}_{R} \\
& +\left(\tilde{\nu}_{R}^{*} \mathbf{b}_{\nu} \mathbf{m}_{M} \tilde{\nu}_{R}^{*}+\tilde{L}^{\top} i \sigma_{2} H_{u} \mathbf{Y}_{\nu} \mathbf{A}_{\nu} \tilde{\nu}_{R}^{*}+\text { H.c. }\right),
\end{aligned}
$$

where $\mathcal{L}_{\text {soft }}^{\text {MSSM }}$ denotes the usual soft SUSY-breaking contribution from the MSSM, and $\mathbf{m}_{\tilde{\nu}}^{2}, \mathbf{b}_{\nu}$ and $\mathbf{A}_{\nu}$ are $n_{R} \times n_{R^{-}}$ dimensional matrices.

A minimal radiative seesaw scenario of the Type $I$ is the ISS realization presented in [33]. As in the standard ISS model $[29,30]$, the radiative model has new fermionic singlets that come in $n$ pairs, i.e., $n_{R}=2 n$. Thus, the model contains $n$ right-handed neutrinos $\nu_{i R}$, with $i=1,2, \ldots, n$, while the remaining $n$ right-handed neutrinos are renamed as $\nu_{i R} \equiv\left(S_{\alpha L}\right)^{C}$, with $i=n+\alpha=n+1$, $n+2, \ldots, 2 n$. In terms of the fields $\left(\nu_{1,2,3 L}, \nu_{i R}^{C}, S_{\alpha L}\right)$, with $i, \alpha=1,2, \ldots, n$, the tree-level neutrino-mass Lagrangian after SSB reads

$$
\begin{aligned}
-\mathcal{L}_{\text {mass }}^{\nu}= & \frac{1}{2}\left(\bar{\nu}_{L}^{C}, \bar{\nu}_{R}, \bar{S}_{L}^{C}\right)\left(\begin{array}{ccc}
\mathbf{0}_{3} & \mathbf{M}_{D} & \mathbf{0}_{3 \times n} \\
\mathbf{M}_{D}^{\top} & \boldsymbol{\mu}_{R} & \mathbf{M}_{N}^{\top} \\
\mathbf{0}_{n \times 3} & \mathbf{M}_{N} & \boldsymbol{\mu}_{S}
\end{array}\right)\left(\begin{array}{c}
\nu_{L} \\
\nu_{R}^{C} \\
S_{L}
\end{array}\right) \\
& + \text { H.c., }
\end{aligned}
$$

with $\mathbf{M}_{D}=\frac{v_{u}}{\sqrt{2}} \mathbf{Y}_{\nu}$. The standard seesaw matrix of the $\nu_{R} \mathrm{SM}$ is recovered if the following identifications are made:

$$
\mathbf{m}_{D} \equiv\left(\mathbf{M}_{D}, \mathbf{0}_{3 \times n}\right), \quad \mathbf{m}_{M} \equiv\left(\begin{array}{cc}
\boldsymbol{\mu}_{R} & \mathbf{M}_{N}^{\top} \\
\mathbf{M}_{N} & \boldsymbol{\mu}_{S}
\end{array}\right) .
$$

The mass matrix of (2.5) has two soft LNV matrix-valued parameters, $\boldsymbol{\mu}_{R, S}$. In the limit where lepton number is preserved $\boldsymbol{\mu}_{R, S}=\mathbf{0}_{n}$, the neutrino mass eigenstates become exact Dirac states to all loop orders. The usual ISS scenario, which has been studied widely in the literature [44,55-58], is obtained for $\boldsymbol{\mu}_{S} \neq \mathbf{0}_{n}$ and $\boldsymbol{\mu}_{R}=\mathbf{0}_{n}$. However, our interest here is the SUSY extension of the radiative ISS model in [33], for which $\boldsymbol{\mu}_{S}=\mathbf{0}_{n}$ and $\boldsymbol{\mu}_{R} \neq \mathbf{0}_{n}$.

In the context of the $\nu_{R}$ MSSM, left and right-handed sneutrinos mix. In the weak basis $\left(\tilde{\nu}_{L}, \tilde{\nu}_{R}^{*}, \tilde{\nu}_{L}^{*}, \tilde{\nu}_{R}\right)^{\top}$, the following sneutrino mass matrix may be derived:

$$
\mathbf{M}_{\tilde{\nu}}^{2}=\left(\begin{array}{cccc}
\mathbf{H}_{1} & \mathbf{N} & \mathbf{0} & \mathbf{M} \\
\mathbf{N}^{\dagger} & \mathbf{H}_{2}^{\top} & \mathbf{M}^{\top} & \mathbf{B}_{\nu}^{\dagger} \\
\mathbf{0} & \mathbf{M}^{*} & \mathbf{H}_{1}^{\top} & \mathbf{N}^{*} \\
\mathbf{M}^{\dagger} & \mathbf{B}_{\nu} & \mathbf{N}^{\top} & \mathbf{H}_{2}
\end{array}\right),
$$

where

$$
\begin{aligned}
\mathbf{H}_{1} & =\mathbf{m}_{\tilde{e}}^{2}+\mathbf{m}_{D}^{*} \mathbf{m}_{D}^{\top}+\frac{1}{2} M_{Z}^{2} \cos 2 \beta \mathbf{1}_{3}, \\
\mathbf{H}_{2} & =\mathbf{m}_{\tilde{\nu}}^{2}+\mathbf{m}_{D}^{\top} \mathbf{m}_{D}^{*}+\mathbf{m}_{M} \mathbf{m}_{M}^{\dagger}, \\
\mathbf{M} & =\mathbf{m}_{D}^{*}\left(\mathbf{A}_{\nu}^{*}-\mu \cot \beta \mathbf{1}_{n_{R}}\right), \\
\mathbf{N} & =\mathbf{m}_{D}^{*} \mathbf{m}_{M}, \\
\mathbf{B}_{\nu} & =\mathbf{b}_{\nu} \mathbf{m}_{M} .
\end{aligned}
$$

Note that the sneutrino mass matrix for the ISS scenario, in which the fields $\tilde{S}_{L}$ are included in conjunction with $\tilde{\nu}_{R}^{*}$, can straightforwardly be obtained after making the identifications for the mass matrices $\mathbf{m}_{M}$ and $\mathbf{m}_{D}$ mentioned above [cf. (2.6)].

\section{RADIATIVE NEUTRINO MASSES}

In this section, we calculate the relevant Feynmandiagrammatic contributions to the neutrino self-energy matrix, $\Sigma(\not p)$. The calculation is carried out in the flavor and weak bases, rather in the mass basis, in order to avoid the use of large dimensional matrices describing neutralino and sneutrino mixings. This enables us to have good control of the soft SUSY-screening phenomenon mentioned in the Introduction. 
For theories with Majorana fermions that we have been considering here, the neutrino self-energy matrix assumes the general form $[59,60]$ :

$$
\begin{aligned}
\Sigma(\not p)= & \Sigma_{L}\left(p^{2}\right) \not p P_{L}+\Sigma_{R}\left(p^{2}\right) \not p P_{R}+\Sigma_{M}\left(p^{2}\right) P_{L} \\
& +\Sigma_{M}^{*}\left(p^{2}\right) P_{R},
\end{aligned}
$$

with $\Sigma_{L, R}\left(p^{2}\right)=\Sigma_{L, R}^{\dagger}\left(p^{2}\right), \Sigma_{L}\left(p^{2}\right)=\Sigma_{R}^{*}\left(p^{2}\right)$ and $\Sigma_{M}\left(p^{2}\right)=$ $\Sigma_{M}^{\top}\left(p^{2}\right)$. Since our interest lies only in computing the left-handed effective neutrino mass matrix $\mathbf{M}_{\nu_{L}}$, which is generated radiatively, this can easily be determined by

$$
\mathbf{M}_{\nu_{L}} P_{L}=-\left.P_{L} \Sigma(\not p) P_{L}\right|_{p \rightarrow 0}=-\Sigma_{M}(0) P_{L} .
$$

In the weak and flavor spaces, the leading-order contributions to $\Sigma_{M}(0)$ are shown in Figs. 1-3. Note that chirality flipping insertions are denoted with a cross on a fermion line. If this chirality flip happens to violate the lepton number as well, we indicate this with a cross inside a circle. In particular, the analytic results derived from the weakand flavor-space diagrams have the advantage that they only depend on the parameters of the Lagrangian and the particle masses, but not on mixing matrix elements.

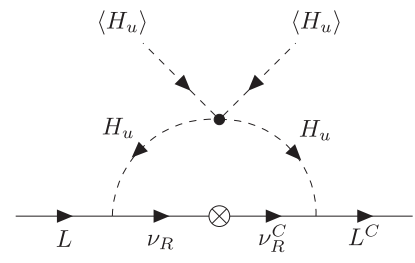

(a)

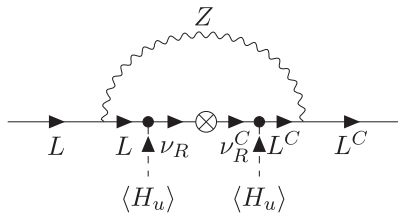

(b)
FIG. 1. Leading-order diagrams in the $\nu_{R} \mathrm{SM}$ that contribute to neutrino masses. An encircled cross indicates a LNV insertion, a heavy dot in diagram (a) stands for $\lambda_{\text {eff }}$ [cf. (3.5)], while a dot in diagram (b) represents an $\mathbf{m}_{D}$ insertion [cf. (2.8c)]. Note that the arrows on the Higgs-propagator lines indicate hypercharge flow.

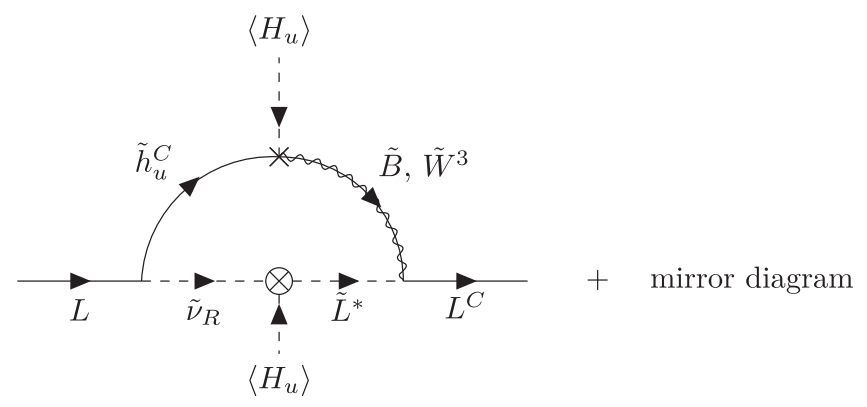

FIG. 2. Supersymmetric one-loop contributions to neutrino masses. The cross represents a chirality flip in the Higgsinogaugino fermionic line, and the encircled cross represents an $F$-term LNV mass insertion [cf. (2.8d)]. (a)

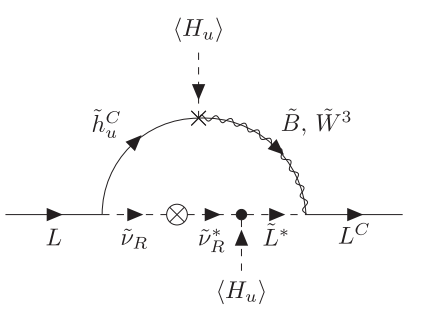

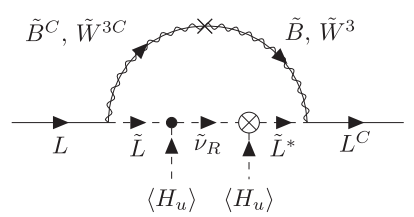

(b)

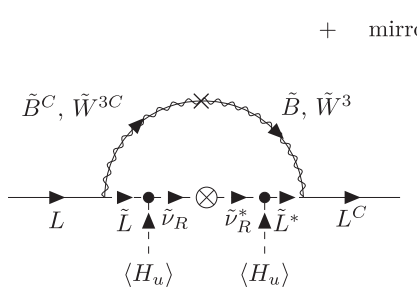

(c) (d)
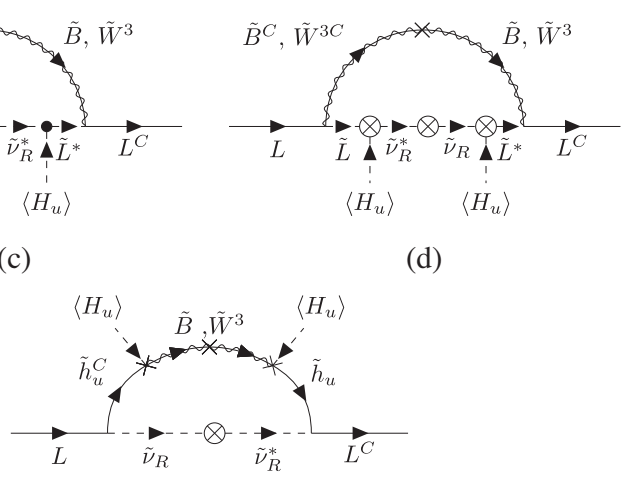

(e)

FIG. 3. Feynman diagrams contributing to neutrino masses when SUSY is softly broken. Note that the right-handed sneutrino propagator has a LNV mass insertion proportional to $\mathbf{B}_{\nu}$.

To avoid too large effects of charged lepton flavor violation, we assume that the soft SUSY-breaking bilinear and trilinear parameters are universal at some low-energy scale, i.e.,

$$
\begin{array}{ll}
\mathbf{m}_{\tilde{e}}^{2}=m_{\tilde{e}}^{2} \mathbf{1}_{3}, & \mathbf{m}_{\tilde{\ell}}^{2}=m_{\tilde{\ell}}^{2} \mathbf{1}_{3}, \\
\mathbf{m}_{\tilde{\nu}}^{2}=m_{\tilde{\nu}}^{2} \mathbf{1}_{n_{R}}, & \mathbf{A}_{\nu}=A_{\nu} \mathbf{1}_{n_{R}},
\end{array}
$$

so that we can ignore renormalization-group effects for simplicity. Moreover, we work in second order approximation for the neutrino Yukawa matrix $\mathbf{Y}_{\nu}$. This is a good approximation provided the mixing between light and heavy neutrinos is reasonably small. To simplify matters, our computation is based on the working hypothesis that the bilinear mass matrix $\mathbf{b}_{\nu}$ is universal,

$$
\mathbf{b}_{\nu}=b_{\nu} \mathbf{1}_{n_{R}} .
$$

It should be noted here that although the final analytic expression $\Sigma_{M}(0)$ is gauge-fixing parameter independent, the computation of the individual diagrams is done in the Landau gauge.

Let us first consider the Feynman graphs shown in Fig. 1 which are closely related to those evaluated in the $\nu_{R} S M$ [31]. At the one-loop level, there are two contributions that involve: (a) the up-type Higgs-boson $H_{u}$ and (b) the $Z$ boson. For the diagram in Fig. 1(a), we must notice that it 
receives significant quantum corrections beyond the oneloop level, through the operator $\lambda_{\mathrm{eff}}\left(H_{u}^{\dagger} H_{u}\right)^{2}$ whose coupling $\lambda_{\text {eff }}$ gets considerably enhanced [61-63] beyond its SUSY tree-level value, i.e., $\lambda_{\text {tree }}=\left(g^{2}+g^{\prime 2}\right) / 8$. The effective coupling $\lambda_{\text {eff }}$ helps to raise the value of the lightest $C P$ even Higgs mass, $m_{h}$, to that of the observed SM-like Higgs resonance, i.e., $m_{h} \approx 125 \mathrm{GeV}$ [64]. For instance, for moderate values of $\tan \beta$, e.g., $\tan \beta \lesssim 20$, one-loop scalar top $(\tilde{t})$ effects become dominant, leading to an effective coupling (see, e.g., [65]),

$$
\begin{aligned}
\lambda_{\text {eff }}= & \lambda_{\text {tree }}\left[1-\frac{3}{8 \pi^{2}} \ln \left(\frac{M_{\tilde{t}}^{2}}{\bar{m}_{t}^{2}}\right)\right] \\
& +\frac{3 y_{t}^{4}}{16 \pi^{2}}\left[\ln \left(\frac{M_{\tilde{t}}^{2}}{\bar{m}_{t}^{2}}\right)+\frac{\left|A_{t}\right|^{2}}{M_{\tilde{t}}^{2}}\left(1-\frac{\left|A_{t}\right|^{2}}{12 M_{\tilde{t}}^{2}}\right)\right],
\end{aligned}
$$

where $\bar{m}_{t}$ is the top-quark pole mass, $y_{t}$ is the top-quark Yukawa coupling, $M_{\tilde{t}}^{2}$ is the average of the soft scalar-top masses squared, and $A_{t}$ is the respective soft trilinear coupling. In the present study, we will not specify the full soft SUSY-breaking sector of the $\nu_{R}$ MSSM, but only adjust $\lambda_{\text {eff }}$, so as to have $m_{h}=125.38 \pm 0.14 \mathrm{GeV}$ [cf. Table II].
TABLE II. Input parameters for our baseline benchmark scenario in the $\nu_{R}$ MSSM.

\begin{tabular}{lc}
\hline \hline Parameter & Numerical value/interval \\
\hline $\tan \beta$ & 2,20 \\
$\mu$ & $1200 \mathrm{GeV}$ \\
$M_{1}$ & $1500 \mathrm{GeV}$ \\
$M_{2}$ & $1500 \mathrm{GeV}$ \\
$m_{h}$ & $125.38 \pm 0.14 \mathrm{GeV}$ \\
$m_{A}$ & $5000 \mathrm{GeV}$ \\
$m_{H}$ & $5002.8 \mathrm{GeV}$ \\
$m_{N}$ & $500 \mathrm{GeV}$ \\
$m_{\tilde{\ell}}^{2}$ & $(3500 \mathrm{GeV})^{2}$ \\
$\mu_{R}$ & {$\left[10^{-6}, 10^{2}\right] \mathrm{GeV}$} \\
\hline \hline
\end{tabular}

This can always be achieved by an appropriate choice of the soft parameters associated with the scalar quark sector of the MSSM [66-68]. Further discussion is given in Appendix A.

Taking the above into account, as well as the pertinent $H_{u}^{*} H_{u}^{*}$ entry of the Higgs-boson propagator matrix (see Appendix B for more details), the $H_{u}$-mediated graph in Fig. 1(a) is found to be

$$
i \Sigma_{M}^{[1(a)]}=2 \lambda_{\mathrm{eff}} \mathbf{m}_{D} \mathbf{m}_{M}^{\dagger} \int \frac{d^{d} k}{(2 \pi)^{d}} \frac{\left(k^{2}+m_{A}^{2} \cos 2 \beta\right)^{2}}{k^{2}\left(k^{2} \mathbf{1}_{n_{R}}-\mathbf{m}_{M} \mathbf{m}_{M}^{\dagger}\right)\left(k^{2}-m_{A}^{2}\right)\left(k^{2}-m_{h}^{2}\right)\left(k^{2}-m_{H}^{2}\right)} \mathbf{m}_{D}^{\top},
$$

where $\lambda_{\text {eff }}$ is the effective quartic coupling defined in (3.5).

Since all loop integrals are evaluated at zero external momentum, it becomes more convenient to express them in terms of the functions

$$
I_{n}\left(m_{1}^{2}, m_{2}^{2}, \ldots, m_{n}^{2}\right) \equiv \int \frac{d^{d} k}{(2 \pi)^{d}} \prod_{j=1}^{n} \frac{1}{k^{2}-m_{j}^{2}}
$$

The functions $I_{n}$ satisfy the recursive relations

$$
\begin{aligned}
& I_{n}\left(m_{1}^{2}, m_{2}^{2}, \ldots, m_{n}^{2}\right) \\
& \quad=\frac{I_{n-1}\left(m_{1}^{2}, m_{3}^{2}, \ldots, m_{n}^{2}\right)-I_{n-1}\left(m_{2}^{2}, m_{3}^{2}, \ldots, m_{n}^{2}\right)}{m_{1}^{2}-m_{2}^{2}} .
\end{aligned}
$$

They are related to the usual Veltman functions [69] by a multiplicative factor. For instance, for $n=2$, we have

$$
I_{2}\left(m_{1}^{2}, m_{2}^{2}\right)=\frac{i}{16 \pi^{2}} B_{0}\left(0, m_{1}^{2}, m_{2}^{2}\right)
$$

where in $d=4-2 \epsilon$ dimensions,

$$
B_{0}\left(0, m_{1}^{2}, m_{2}^{2}\right)=C_{\mathrm{UV}}-\frac{m_{1}^{2}}{m_{1}^{2}-m_{2}^{2}} \ln \left(\frac{m_{1}^{2}}{m_{2}^{2}}\right)-\ln \left(\frac{m_{2}^{2}}{\mu^{2}}\right)+1 \text {. }
$$

Here, $C_{\mathrm{UV}}=\frac{2}{\epsilon}+\ln 4 \pi-\gamma_{\mathrm{E}}$ is an UV constant, $\gamma_{\mathrm{E}}$ is the Euler-Mascheroni constant, and $\mu$ is an arbitrary mass scale introduced by 't Hooft. As we will see below, all loop contributions yield combinations of functions $I_{n}$, with $n \geq 3$. In light of (3.8), this means that the UV divergent parts depending on $C_{\mathrm{UV}}$ cancel out completely, as required by the renormalizability of the theory.

By virtue of the $I_{n}$ functions defined in (3.7), we may now reexpress (3.6) as follows:

$$
\begin{aligned}
i \Sigma_{M}^{[1(a)]}= & 2 \lambda_{\mathrm{eff}} \mathbf{m}_{D} \mathbf{m}_{M}^{\dagger}\left[I_{3}\left(m_{h}^{2}, m_{H}^{2}, \mathbf{m}_{M} \mathbf{m}_{M}^{\dagger}\right)\right. \\
& +m_{A}^{2}(1+2 \cos 2 \beta) I_{4}\left(m_{A}^{2}, m_{h}^{2}, m_{H}^{2}, \mathbf{m}_{M} \mathbf{m}_{M}^{\dagger}\right) \\
& \left.+m_{A}^{4} \cos ^{2} 2 \beta I_{5}\left(0, m_{A}^{2}, m_{h}^{2}, m_{H}^{2}, \mathbf{m}_{M} \mathbf{m}_{M}^{\dagger}\right)\right] \mathbf{m}_{D}^{\top} .
\end{aligned}
$$

Likewise, the $Z$-boson mediated diagram in Fig. 1(b) is given by 
$i \Sigma_{M}^{[1(b)]}=\frac{3}{4}\left(g^{2}+g^{\prime 2}\right) \mathbf{m}_{D} \mathbf{m}_{M}^{\dagger} I_{3}\left(0, M_{Z}^{2}, \mathbf{m}_{M} \mathbf{m}_{M}^{\dagger}\right) \mathbf{m}_{D}^{\top}$.

Let us now turn our attention to the respective supersymmetric contributions to radiative neutrino masses shown in Fig. 2. The evaluation of these graphs can be done using the neutralino propagator matrix in the weak basis, by following the procedure outlined in Appendix B. Thus, the loop integral for the SUSY graphs in Fig. 2 reads

$$
\begin{aligned}
i \Sigma_{M}^{[2]}= & \frac{1}{v_{u}} \int \frac{d^{d} k}{(2 \pi)^{d}} \frac{1}{k^{2} \mathbf{1}_{3}-\tilde{\mathbf{H}}_{1}^{\top}} \mathbf{N}^{*} \frac{1}{k^{2} \mathbf{1}_{n_{R}}-\tilde{\mathbf{H}}_{2}} \mathbf{m}_{D}^{\top} \frac{g\left(M_{\chi^{0}} \operatorname{adj} \mathcal{F}\right)_{\tilde{h}_{u}^{0} \tilde{W}^{3}}-g^{\prime}\left(M_{\chi^{0}} \operatorname{adj} \mathcal{F}\right)_{\tilde{h}_{u}^{0} \tilde{B}}}{\operatorname{det} \mathcal{F}} \\
& + \text { transpose, }
\end{aligned}
$$

where $\mathcal{F}$ is a $4 \times 4$ matrix related to the weak-space neutralino propagator and adj $\mathcal{F}$ stands for its adjunct. More details may be found in Appendix B. In (3.13), we defined $\left.\tilde{\mathbf{H}}_{1,2} \equiv \mathbf{H}_{1,2}\right|_{\mathbf{m}_{D}=\mathbf{0}}$, consistent with our second-order expansion in the neutrino Yukawa matrix $\mathbf{Y}_{\nu}$. Since $\tilde{\mathbf{H}}_{\mathbf{1}}$ is proportional to the identity in the same order of approximation, we may write $\tilde{\mathbf{H}}_{1}^{\top}=\tilde{H}_{1}^{*} \mathbf{1}_{3}$. Taking the above simplifications into account, the loop integral may successively be evaluated as follows:

$$
\begin{aligned}
i \Sigma_{M}^{[2]}= & \frac{1}{v_{u}} \mathbf{N}^{*} \int \frac{d^{d} k}{(2 \pi)^{d}} \frac{g\left(M_{\chi^{0}} \operatorname{adj} \mathcal{F}\right)_{\tilde{h}_{u}^{0}} \tilde{W}^{3}-g^{\prime}\left(M_{\chi^{0}} \operatorname{adj} \mathcal{F}\right)_{\tilde{h}_{u}^{0} \tilde{B}}}{\operatorname{det} \mathcal{F}\left(k^{2}-\tilde{H}_{1}^{*}\right)\left(k^{2} \mathbf{1}_{n_{R}}-\tilde{\mathbf{H}}_{2}\right)}+\text { transpose } \\
= & \mathbf{m}_{D} \mathbf{m}_{M}^{\dagger} \int \frac{d^{d} k}{(2 \pi)^{d}} \frac{A k^{6}+B k^{4}+C k^{2}+D}{\operatorname{det} \mathcal{F}\left(k^{2}-\tilde{H}_{1}^{*}\right)\left(k^{2} \mathbf{1}_{n_{R}}-\tilde{\mathbf{H}}_{2}\right)} \mathbf{m}_{D}^{\top}+\text { transpose } \\
= & \mathbf{m}_{D} \mathbf{m}_{M}^{\dagger}\left[A I_{3}\left(\tilde{H}_{1}^{*}, \tilde{\mathbf{H}}_{2}, m_{\chi_{4}^{0}}^{2}\right)+\mathcal{B}_{A, B} I_{4}\left(\tilde{H}_{1}^{*}, \tilde{\mathbf{H}}_{2}, m_{\chi_{3}^{0}}^{2}, m_{\chi_{4}^{0}}^{2}\right)\right. \\
& \left.+\mathcal{C}_{A, B, C} I_{5}\left(\tilde{H}_{1}^{*}, \tilde{\mathbf{H}}_{2}, m_{\chi_{2}^{0}}^{2}, m_{\chi_{3}^{0}}^{2}, m_{\chi_{4}^{0}}^{2}\right)+\mathcal{D}_{A, B, C, D} I_{6}\left(\tilde{H}_{1}^{*}, \tilde{\mathbf{H}}_{2}, m_{\chi_{1}^{0}}^{2}, m_{\chi_{2}^{0}}^{2}, m_{\chi_{3}^{0}}^{2}, m_{\chi_{4}^{0}}^{2}\right)\right] \mathbf{m}_{D}^{\top}+\text { transpose, }
\end{aligned}
$$

with

$$
\begin{aligned}
\mathcal{B}_{A, B} & =A\left(m_{\chi_{1}^{0}}^{2}+m_{\chi_{2}^{0}}^{2}+m_{\chi_{3}^{0}}^{2}\right)+B, \\
\mathcal{C}_{A, B, C} & =A\left(m_{\chi_{1}^{0}}^{4}+m_{\chi_{1}^{0}}^{2} m_{\chi_{2}^{0}}^{2}+m_{\chi_{2}^{0}}^{4}\right)+B\left(m_{\chi_{1}^{0}}^{2}+m_{\chi_{2}^{0}}^{2}\right)+C, \\
\mathcal{D}_{A, B, C, D} & =A m_{\chi_{1}^{0}}^{6}+B m_{\chi_{1}^{0}}^{4}+C m_{\chi_{1}^{0}}^{2}+D .
\end{aligned}
$$

Note that the analytic forms of the coefficients $A, B, C$, and $D$ are given in $(\mathrm{C} 2)$.

It can now be verified that the sum of the self-energy expressions in (3.11), (3.12), and (3.14) vanishes in the exact SUSY limit, which is realized for $\tan \beta=1, \mu=0$, and $M_{\text {SUSY }} \rightarrow 0$, with $\lambda_{\text {eff }} \rightarrow \lambda_{\text {tree }}$, according to our discussion in Appendix A.

Finally, we must take into consideration weak- and flavor-space graphs, as depicted in Fig. 3, which are generated when SUSY is softly broken. We start by calculating the loop integral for the diagram in Fig. 3(a). This is given by

$$
\begin{aligned}
i \Sigma_{M}^{[3(a)]}= & \frac{1}{v_{u}} \int \frac{d^{d} k}{(2 \pi)^{d}} \frac{1}{k^{2} \mathbf{1}_{3}-\tilde{\mathbf{H}}_{\mathbf{1}}^{\top}} \mathbf{M}^{*} \frac{1}{k^{2} \mathbf{1}_{n_{R}}-\tilde{\mathbf{H}}_{\mathbf{2}}^{\top}} b_{\nu}^{*} \mathbf{m}_{M}^{\dagger} \frac{1}{k^{2} \mathbf{1}_{n_{R}}-\tilde{\mathbf{H}}_{\mathbf{2}}} \mathbf{m}_{D}^{\top} \frac{g\left(M_{\chi^{0}} \operatorname{adj} \mathcal{F}\right) \tilde{h}_{u}^{0} \tilde{W}^{3}-g^{\prime}\left(M_{\chi^{0}} \operatorname{adj} \mathcal{F}\right) \tilde{h}_{u}^{0} \tilde{B}}{\operatorname{det} \mathcal{F}} \\
& + \text { transpose. }
\end{aligned}
$$

We may now employ the identity,

$$
f\left(\mathbf{m}_{M}^{\dagger} \mathbf{m}_{M}\right) \mathbf{m}_{M}^{\dagger}=\mathbf{m}_{M}^{\dagger} f\left(\mathbf{m}_{M} \mathbf{m}_{M}^{\dagger}\right)
$$

which holds for an arbitrary regular function $f(x)$, in order to rewrite (3.16) as follows:

$$
\begin{aligned}
i \Sigma_{M}^{[3(a)]} & =\frac{1}{v_{u}} \mathbf{M}^{*} b_{\nu}^{*} \mathbf{m}_{M}^{\dagger} \int \frac{d^{d} k}{(2 \pi)^{d}} \frac{g\left(M_{\chi^{0}} \operatorname{adj} \mathcal{F}\right)_{\tilde{h}_{u}^{0}} \tilde{W}^{3}-g^{\prime}\left(M_{\chi^{0}} \operatorname{adj} \mathcal{F}\right)_{\tilde{h}_{u}^{0} \tilde{B}}}{\operatorname{det} \mathcal{F}\left(k^{2}-\tilde{H}_{1}^{*}\right)\left(k^{2} \mathbf{1}_{n_{R}}-\tilde{\mathbf{H}}_{2}\right)^{2}}+\text { transpose } \\
& =\mathbf{M}^{*} b_{\nu}^{*} \mathbf{m}_{M}^{\dagger} \int \frac{d^{d} k}{(2 \pi)^{d}} \frac{A k^{6}+B k^{4}+C k^{2}+D}{\operatorname{det} \mathcal{F}\left(k^{2}-\tilde{H}_{1}^{*}\right)\left(k^{2} \mathbf{1}_{n_{R}}-\tilde{\mathbf{H}}_{2}\right)^{2}} \mathbf{m}_{D}^{\top}+\text { transpose. }
\end{aligned}
$$


Following the same procedure as before, we eventually arrive at

$$
\begin{aligned}
i \Sigma_{M}^{[3(a)]}= & b_{\nu}^{*} \mathbf{m}_{D}\left(\mathbf{A}_{\nu}-\mu^{*} \cot \beta \mathbf{1}_{n_{R}}\right) \mathbf{m}_{M}^{\dagger}\left[A I_{4}\left(\tilde{H}_{1}^{*}, \tilde{\mathbf{H}}_{2}, \tilde{\mathbf{H}}_{2}, m_{\chi_{4}^{0}}^{2}\right)\right. \\
& +\mathcal{B}_{A, B} I_{5}\left(\tilde{H}_{1}^{*}, \tilde{\mathbf{H}}_{2}, \tilde{\mathbf{H}}_{2}, m_{\chi_{3}^{0}}^{2}, m_{\chi_{4}^{0}}^{2}\right)+\mathcal{C}_{A, B, C} I_{6}\left(\tilde{H}_{1}^{*}, \tilde{\mathbf{H}}_{2}, \tilde{\mathbf{H}}_{2}, m_{\chi_{2}^{0}}^{2}, m_{\chi_{3}^{0}}^{2}, m_{\chi_{4}^{0}}^{2}\right) \\
& \left.+\mathcal{D}_{A, B, C, D} I_{7}\left(\tilde{H}_{1}^{*}, \tilde{\mathbf{H}}_{2}, \tilde{\mathbf{H}}_{2}, m_{\chi_{1}^{0}}^{2}, m_{\chi_{2}^{0}}^{2}, m_{\chi_{3}^{0}}^{2}, m_{\chi_{4}^{0}}^{2}\right)\right] \mathbf{m}_{D}^{\top}+\text { transpose }
\end{aligned}
$$

Here, the index-valued functions $\mathcal{B}_{A, B}, \mathcal{C}_{A, B, C}$, and $\mathcal{D}_{A, B, C, D}$ are given in (3.15).

The diagram in Fig. 3(b) can be evaluated in a similar manner, involving the entries $\tilde{B} \tilde{B}$ and $\tilde{W}^{3} \tilde{W}^{3}$ of the neutralino propagator matrix. More explicitly, we find

$$
\begin{aligned}
i \Sigma_{M}^{[3(b)]}= & \mathbf{m}_{D}\left(\mathbf{A}_{\nu}-\mu^{*} \cot \beta \mathbf{1}_{n_{R}}\right) \mathbf{m}_{M}^{\dagger}\left[A^{\prime} I_{4}\left(\tilde{H}_{1}, \tilde{H}_{1}^{*}, \tilde{\mathbf{H}}_{2}, m_{\chi_{4}^{0}}^{2}\right)\right. \\
& +\mathcal{B}_{A^{\prime}, B^{\prime}} I_{5}\left(\tilde{H}_{1}, \tilde{H}_{1}^{*}, \tilde{\mathbf{H}}_{2}, m_{\chi_{3}^{0}}^{2}, m_{\chi_{4}^{0}}^{2}\right)+\mathcal{C}_{A^{\prime}, B^{\prime}, C^{\prime}} I_{6}\left(\tilde{H}_{1}, \tilde{H}_{1}^{*}, \tilde{\mathbf{H}}_{2}, m_{\chi_{1}^{0}}^{2}, m_{\chi_{3}^{0}}^{2}, m_{\chi_{4}^{0}}^{2}\right) \\
& \left.+\mathcal{D}_{A^{\prime}, B^{\prime}, C^{\prime}, D^{\prime}} I_{7}\left(\tilde{H}_{1}, \tilde{H}_{1}^{*}, \tilde{\mathbf{H}}_{2}, m_{\chi_{1}^{0}}^{2}, m_{\chi_{2}^{0}}^{2}, m_{\chi_{3}^{0}}^{2}, m_{\chi_{4}^{0}}^{2}\right)\right] \mathbf{m}_{D}^{\top}+\text { transpose }
\end{aligned}
$$

where the index-valued functions $\mathcal{B}_{A^{\prime}, B^{\prime}}, \mathcal{C}_{A^{\prime}, B^{\prime}, C^{\prime}}$, and $\mathcal{D}_{A^{\prime}, B^{\prime}, C^{\prime}, D^{\prime}}$ are determined through (3.15), and the coefficients $A^{\prime}, B^{\prime}$, $C^{\prime}$, and $D^{\prime}$ are computed in (C.4).

The remaining Feynman-diagrammatic contributions shown in Figs. 3(c)-(e) are respectively given by

$$
\begin{aligned}
i \Sigma_{M}^{[3(c)]}= & b_{\nu}^{*} \mathbf{m}_{D}\left(\mathbf{A}_{\nu}-\mu^{*} \cot \beta \mathbf{1}_{n_{R}}\right) \mathbf{m}_{M}^{\dagger}\left[A^{\prime} I_{5}\left(\tilde{H}_{1}, \tilde{H}_{1}^{*}, \tilde{\mathbf{H}}_{2}, \tilde{\mathbf{H}}_{2}, m_{\chi_{4}^{0}}^{2}\right)\right. \\
& +\mathcal{B}_{A^{\prime}, B^{\prime}} I_{6}\left(\tilde{H}_{1}, \tilde{H}_{1}^{*}, \tilde{\mathbf{H}}_{2}, \tilde{\mathbf{H}}_{2}, m_{\chi_{3}^{0}}^{2}, m_{\chi_{4}^{0}}^{2}\right)+\mathcal{C}_{A^{\prime}, B^{\prime}, C^{\prime}} I_{7}\left(\tilde{H}_{1}, \tilde{H}_{1}^{*}, \tilde{\mathbf{H}}_{2}, \tilde{\mathbf{H}}_{2}^{\prime} m_{\chi_{1}^{0}}^{2}, m_{\chi_{3}^{0}}^{2}, m_{\chi_{4}^{0}}^{2}\right) \\
& +\mathcal{D}_{A^{\prime}, B^{\prime}, C^{\prime}, D^{\prime}} I_{8}\left(\tilde{H}_{1}, \tilde{H}_{1}^{*}, \tilde{\mathbf{H}}_{2}, \tilde{\mathbf{H}}_{2}, m_{\chi_{1}^{0}}^{2}, m_{\chi_{2}^{0}}^{2}, m_{\chi_{3}^{0}}^{2}, m_{\chi_{4}^{0}}^{2}\right]\left(\mathbf{A}_{\nu}^{\top}-\mu^{*} \cot \beta \mathbf{1}_{n_{R}}\right) \mathbf{m}_{D}^{\top}, \\
i \Sigma_{M}^{[3(d)]]}= & b_{\nu} \mathbf{m}_{D} \mathbf{m}_{M}^{\dagger} \mathbf{m}_{M}\left[A^{\prime} I_{5}\left(\tilde{H}_{1}, \tilde{H}_{1}^{*}, \tilde{\mathbf{H}}_{2}^{\top}, \tilde{\mathbf{H}}_{2}^{\top}, m_{\chi_{4}^{0}}^{2}\right)\right. \\
& +\mathcal{B}_{A^{\prime}, B^{\prime}} I_{6}\left(\tilde{H}_{1}, \tilde{H}_{1}^{*}, \tilde{\mathbf{H}}_{2}^{\top}, \tilde{\mathbf{H}}_{2}^{\top}, m_{\chi_{3}^{0}}^{2}, m_{\chi_{4}^{0}}^{2}\right)+\mathcal{C}_{A^{\prime}, B^{\prime}, C^{\prime}} I_{7}\left(\tilde{H}_{1}, \tilde{H}_{1}^{*}, \tilde{\mathbf{H}}_{2}^{\top}, \tilde{\mathbf{H}}_{2}^{\top}, m_{\chi_{1}^{0}}^{2}, m_{\chi_{3}^{0}}^{2}, m_{\chi_{4}^{0}}^{2}\right) \\
& \left.+\mathcal{D}_{A^{\prime}, B^{\prime}, C^{\prime}, D^{\prime}} I_{8}\left(\tilde{H}_{1}, \tilde{H}_{1}^{*}, \tilde{\mathbf{H}}_{2}^{\top}, \tilde{\mathbf{H}}_{2}^{\top}, m_{\chi_{1}^{0}}^{2}, m_{\chi_{2}^{0}}^{2}, m_{\chi_{3}^{0}}^{2}, m_{\chi_{4}^{0}}^{2}\right)\right] \mathbf{m}_{M}^{\dagger} \mathbf{m}_{D}^{\top}, \\
i \Sigma_{M}^{[3(e)]}= & b_{\nu}^{*} \mathbf{m}_{D} \mathbf{m}_{M}^{\dagger}\left[A^{\prime \prime} I_{3}\left(\tilde{\mathbf{H}}_{2}, \tilde{\mathbf{H}}_{2}, m_{\chi_{4}^{0}}^{2}\right)+\mathcal{B}_{A^{\prime \prime}, B^{\prime \prime}} I_{4}\left(\tilde{\mathbf{H}}_{2}, \tilde{\mathbf{H}}_{2}, m_{\chi_{3}^{0}}^{2}, m_{\chi_{4}^{0}}^{2}\right)\right. \\
& +\mathcal{C}_{A^{\prime \prime}, B^{\prime \prime}, C^{\prime \prime}} I_{5}\left(\tilde{\mathbf{H}}_{2}, \tilde{\mathbf{H}}_{2}, m_{\chi_{1}^{0}}^{2}, m_{\chi_{3}^{0}}^{2}, m_{\chi_{4}^{0}}^{2}\right) \\
& \left.+\mathcal{D}_{A^{\prime \prime}, B^{\prime \prime}, C^{\prime \prime}, D^{\prime \prime}} I_{6}\left(\tilde{\mathbf{H}}_{2}, \tilde{\mathbf{H}}_{2}, m_{\chi_{1}^{0}}^{2}, m_{\chi_{2}^{0}}^{2}, m_{\chi_{3}^{0}}^{2}, m_{\chi_{4}^{0}}^{2}\right)\right] \mathbf{m}_{D}^{\top} .
\end{aligned}
$$

where the coefficients that $A^{\prime \prime}, B^{\prime \prime}, C^{\prime \prime}$, and $D^{\prime \prime}$ are given in (C.6).

We conclude this section by making a few important remarks. First, we note that the flavor structure of the selfenergy contribution given in (3.20) depends strongly on the texture of $\mathbf{A}_{\nu}$, and it is independent of the sneutrino Majorana mass matrix $\mathbf{b}_{\nu}$. Second, we observe that the last self-energy contribution in (3.23) becomes rather relevant since its flavor structure is quite similar to that obtained by the $\nu_{R} \mathrm{SM}$ graphs in Fig. 1 . It can be roughly of the same order without assuming very high values for the parameter $b_{\nu}$. This allows the existence of a parameter space where the $\nu_{R} \mathrm{SM}$ contributions are screened by this diagram. This fact will be used in the next section for defining appropriate benchmark scenarios for radiative neutrino masses in the $\nu_{R}$ MSSM. Finally, the diagram in Fig. 3(e) is nonzero, even if all soft masses are set to zero, provided the $\mu$ parameter is nonzero. To be specific, there is a SUSY counterpart to Fig. 1(a) involving a single $\mu$-dependent chirality flip on the Higgsino line in the loop. However, this graph contributes with four powers in the neutrino Yukawa matrix $\mathbf{Y}_{\nu}$, and so it can be consistently ignored in the leading second-order approximation of $\mathbf{Y}_{\nu}$ that we have been working here. 


\section{NUMERICAL RESULTS}

We will now perform a numerical analysis for a few representative scenarios that give rise to radiative neutrino masses in the $\nu_{R} \mathrm{MSSM}$. As there are many parameters that can vary independently, we choose the baseline benchmark model exhibited in Table II. In addition, as discussed in the previous section and in Appendix A, we use an effective quartic coupling $\lambda_{\text {eff }}$ for the quadrilinear interaction $\left(H_{u}^{\dagger} H_{u}\right)^{2}$ induced by quantum effects, such that the mass of the $h$ boson is equal to that of the observed SM-like Higgs boson, i.e., $m_{h}=125.38 \pm 0.14 \mathrm{GeV}$. Finally, unless stated otherwise, we assume a universal structure for the soft SUSY-breaking parameters, as given in (3.3).

An important constraint in our numerical analysis is the compatibility of the effective neutrino mass matrix
$\mathbf{M}_{\nu_{L}}=\Sigma_{M}(0)$, obtained through (3.2), with the low-energy neutrino data. To be explicit, we require that

$$
\Sigma_{M}(0)=\mathbf{M}_{\nu_{L}}^{\exp }=U^{\top} \widehat{\mathbf{M}}_{\nu_{L}} U
$$

where $U$ is the Pontecorvo-Maki-Nakagawa-Sakata matrix [70,71], and $\widehat{\mathbf{M}}_{\nu_{L}}=\operatorname{diag}\left(m_{1}, m_{2}, m_{3}\right)$ and $m_{1,2,3}$ are the light neutrino masses. To match our self-energy contributions $\Sigma_{M}$ derived in the previous section to $\mathbf{M}_{\nu_{L}}^{\text {exp }}$, we neglect nonunitarity effects that come from light-to-heavy neutrino mixing and assume that the charged lepton Yukawa matrix $\mathbf{Y}_{l}$ is diagonal and already expressed in the mass basis. With the above assumptions in mind, the matrix $U$ can be parametrized as follows:

$$
U=\left(\begin{array}{ccc}
c_{12} c_{13} & s_{12} c_{13} & s_{13} e^{-i \delta} \\
-s_{12} c_{23}-c_{12} s_{23} s_{13} e^{i \delta} & c_{12} c_{23}-s_{12} s_{23} s_{13} e^{i \delta} & s_{23} c_{13} \\
s_{12} c_{23}-c_{12} c_{23} s_{13} e^{i \delta} & -c_{12} s_{23}-s_{12} c_{23} s_{13} e^{i \delta} & c_{23} c_{13}
\end{array}\right) \times \operatorname{diag}\left(e^{i \alpha_{1} / 2}, e^{i \alpha_{2} / 2}, 1\right)
$$

where $c_{i j}=\cos \theta_{i j}$ and $s_{i j}=\sin \theta_{i j}$ are the neutrino mixing angles, $\delta$ is the so-called Dirac phase, and $\alpha_{1,2}$ are the Majorana phases.

In our numerical estimates, we assume normal neutrino mass ordering. This choice is favored by recent global fits of neutrino oscillation data. For definiteness, we use the latest best fit values for the neutrino oscillation parameters [72]:

$$
\begin{aligned}
& \Delta m_{21}^{2} \equiv m_{2}^{2}-m_{1}^{2}=7.50 \times 10^{-5}(\mathrm{eV})^{2}, \\
& \Delta m_{31}^{2} \equiv m_{3}^{2}-m_{1}^{2}=2.56 \times 10^{-3}(\mathrm{eV})^{2}, \\
& \sin ^{2} \theta_{12}=34.3^{\circ}, \quad \sin ^{2} \theta_{23}=48.79^{\circ}, \\
& \sin ^{2} \theta_{12}=8.58^{\circ}, \quad \delta=216^{\circ} .
\end{aligned}
$$

Furthermore, we set $m_{1}=0$ and $\alpha_{1,2}=0$.

Let us first consider the radiative ISS scenario of the $\nu_{R}$ MSSM described in Sec. II, with $\boldsymbol{\mu}_{R} \neq \mathbf{0}_{n}, \boldsymbol{\mu}_{S}=\mathbf{0}_{n}$, and $n=2$ pairs of singlet neutrinos. This model necessarily implies a massless neutrino, i.e., $m_{1}=0$, at the one-loop level. This should be contrasted to the standard ISS model, with $\boldsymbol{\mu}_{R}=0$ and $\boldsymbol{\mu}_{S} \neq 0$, in which case one pair of singlet neutrinos (i.e., $n=1$ ) would have been sufficient to accommodate the neutrino oscillation data (see, e.g., [44]).

For our illustrative purposes, we will initially assume that the right-handed sneutrino sector is supersymmetric and only consider minimal departures from it, by taking that either $b_{\nu}$ or $A_{\nu}$ is nonzero, but putting $m_{\tilde{\nu}}^{2}=0$ in all settings. For such scenarios, the dominant contributions to the effective neutrino mass matrix $\mathbf{M}_{\nu_{L}}$ arise from the diagrams shown in Figs. 1, 2, and 3(b). For the latter graph, the dominant SUSY effect on $\mathbf{M}_{\nu_{L}}$ comes from the $\mu$ term. In fact, this last effect can screen completely that $\nu_{R} \mathrm{SM}$ contribution from the graphs in Fig. 1, even in the limit of a fully supersymmetric neutrino sector with $b_{\nu}=A_{\nu}=0$.

Following [33], we define the parameter $m_{D}^{\max }=$ $\max \left|\left(\mathbf{M}_{D}\right)_{i j}\right|$, which enables us to introduce the quantity

$$
\lambda_{D}=\left(m_{D}^{\max }\right)^{2} / m_{N}^{2}
$$

Notice that $\lambda_{D}$ quantifies the size of the light-to-heavy neutrino mixing in quadrature.

In Fig. 4, we present exclusion plots of the SUSY parameter $\mu_{R}$ as a function of $\lambda_{D}$, for two scenarios: (i) left panel: $b_{\nu} \neq 0\left(A_{\nu}=0\right)$ and (ii) right panel: $A_{\nu} \neq 0$ $\left(b_{\nu}=0\right)$. In the left panel of Fig. 4, we see an effect of complete cancellation for $b_{\nu}=b_{\nu}^{0}=0.289 \mathrm{GeV}$, when $\tan \beta=2$, and $b_{\nu}=b_{\nu}^{0}=0.399 \mathrm{GeV}$, when $\tan \beta=20$. Also, we see a similar effect to occur in the right panel of Fig. 4 , for $A_{\nu}=A_{\nu}^{0} \simeq 389.1 \mathrm{TeV}$. It is interesting to notice that for large regions of the parameters $b_{\nu}$ and $A_{\nu}$, the allowed values for the LNV parameter $\mu_{R}$ can be nearly four orders of magnitude higher in the $\nu_{R}$ MSSM than in the $\nu_{R} S M$. In fact, the soft SUSY-screening phenomenon can be so strong, that the LNV scale $\mu_{R}$ can get close to the electroweak scale for relatively large light-to-heavy neutrino mixings of order $10^{-2}$, corresponding to $\lambda_{D} \sim 10^{-4}$. Most interestingly, as can be seen from the left panel of Fig. 4 given by the line in red, such a SUSY-screening phenomenon can take place for an exact supersymmetric sneutrino sector, with $b_{\nu}=A_{\nu}=0$, but for an unusually large value of the $\mu$ parameter, $\mu=\mu^{0} \simeq-770.3 \mathrm{TeV}$. 

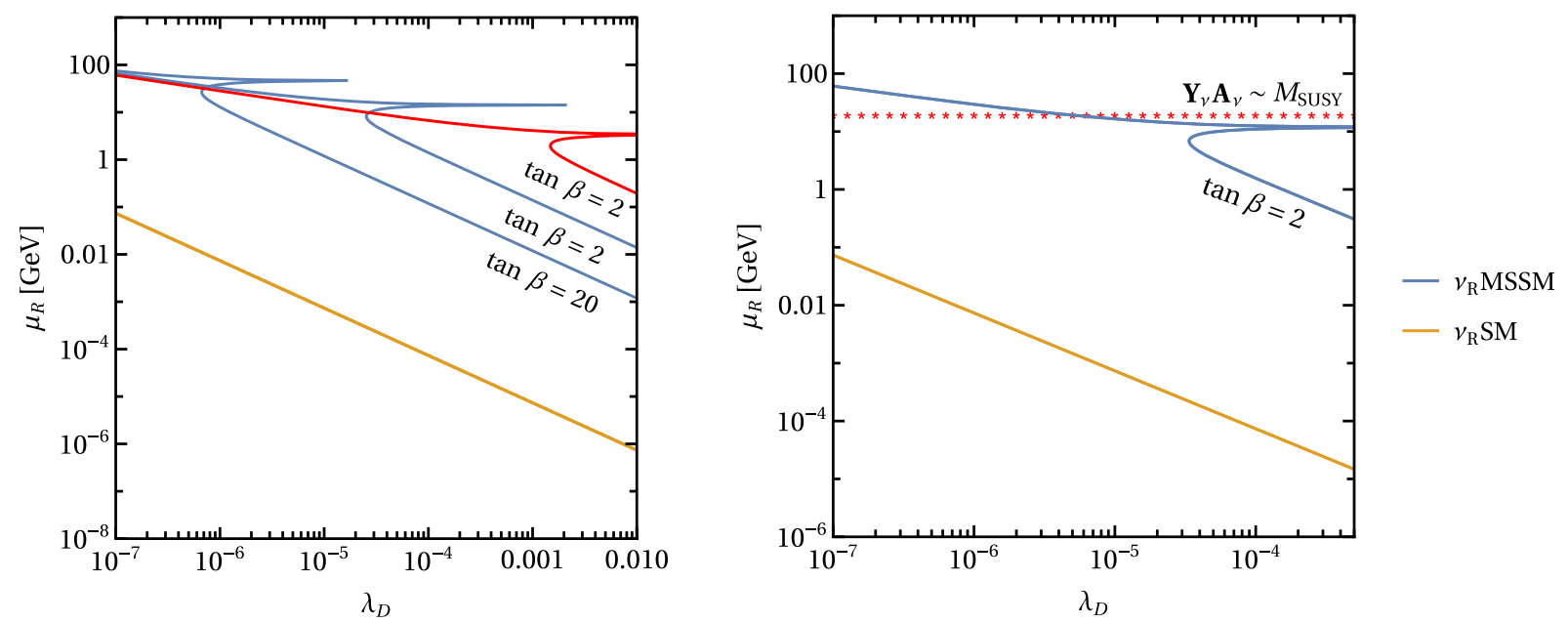

FIG. 4. Numerical estimates of the LNV parameter $\mu_{R}$ versus $\lambda_{D}$ for two scenarios: (i) $b_{\nu} \neq 0$, with $A_{\nu}=0$ (left frame) and (ii) $A_{\nu} \neq 0$, with $b_{\nu}=0$ (right frame). For scenario (i), the screening values for the soft bilinear parameter are: $b_{\nu}^{0}=0.289 \mathrm{GeV}$ for tan $\beta=2$ and $b_{\nu}^{0}=0.399 \mathrm{GeV}$ for $\tan \beta=20$. For scenario (ii), the screening soft trilinear coupling is: $A_{\nu}^{0} \simeq 389.1 \mathrm{TeV}$. The line in red in the left panel shows the predictions obtained from a fully supersymmetric singlet sector, achievable for $\mu=\mu^{0} \simeq-770.3$ TeV. The horizontal dashed line on the right frame indicates the value of $\mu_{R}$, at which the largest entry of $\mathbf{Y}_{\nu} \mathbf{A}_{\nu}$ reaches $M_{\mathrm{SUSY}}$.

This very large value of $\mu$ would require superheavy squark masses of order $10^{3} \mathrm{TeV}$ and higher, as could happen in scenarios of split SUSY [73-75].

In Fig. 5, we display the dependence of the cancelling values $b_{\nu}^{0}$ and $A_{\nu}^{0}$ as functions of the heavy $C P$-even Higgsboson mass $m_{H}$. As can be seen from the left panel in Fig. 5, the $\nu_{R}$ SM-like contribution decreases somewhat as the heavy scalar sector decouples, and as such, a smaller value of $b_{\nu}^{0}$ will be needed to provide the required cancellation. At the larger value of $\tan \beta=20$, the quantum loop effects on the quartic coupling $\left(H_{u}^{\dagger} H_{u}\right)^{2}$ become less pronounced, thus making the diagram in Fig. 1(a) less significant. The same features can be seen in the right panel of Fig. 5, where the dependence of $A_{\nu}^{0}$ on $m_{H}$ is exhibited.

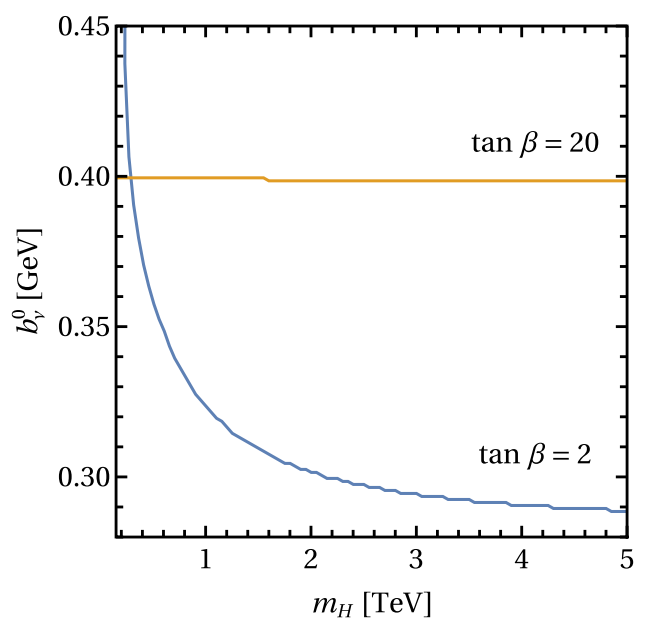

The above SUSY-screening phenomenon may prevent specific flavor structures of $\mathbf{Y}_{\nu}$ that may occur in the $\nu_{R} \mathrm{SM}$ from producing too large finite quantum corrections to light neutrino masses, when the heavy singlet neutrinos happen to be nondegenerate in mass [31]. As an illustrative example, let us consider a three-generation scenario with $n_{R}=3$ right-handed neutrinos. After SSB, the Dirac mass matrix of such a scenario takes on the form

$$
\mathbf{m}_{D}=\frac{v_{u}}{\sqrt{2}}\left(\begin{array}{ccc}
a & b e^{2 i \pi / 3} & c e^{-2 i \pi / 3} \\
a & b e^{2 i \pi / 3} & c e^{-2 i \pi / 3} \\
a & b e^{2 i \pi / 3} & c e^{-2 i \pi / 3}
\end{array}\right),
$$

when expressed in a flavor basis in which the singlet neutrino mass matrix is diagonal and positive, i.e.,

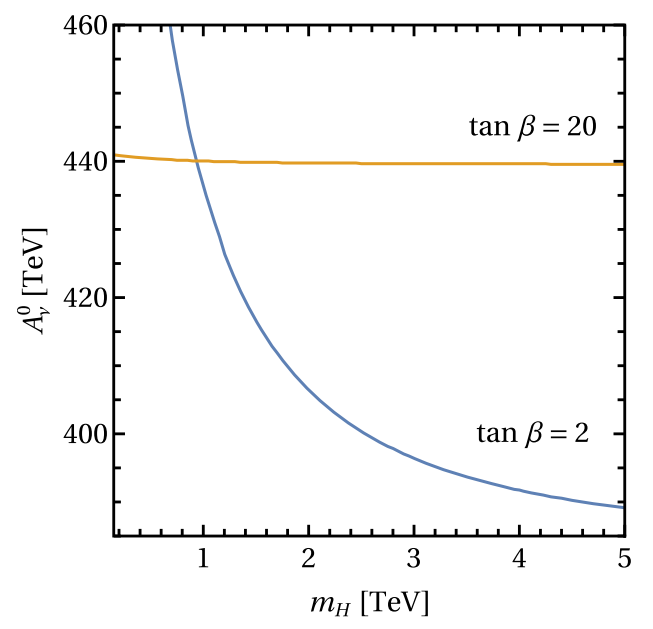

FIG. 5. The dependence of the exact screening values for $b_{\nu}^{0}$ (left frame) and $A_{\nu}^{0}$ (right frame) on the heavy Higgs mass $m_{H}$, after setting $\mu_{R}=10^{-2} \mathrm{GeV}$. 


$$
\mathbf{m}_{M}=\operatorname{diag}\left(m_{N}, m_{N}+\delta_{N}, m_{N}+2 \delta_{N}\right) .
$$

Here, the parameter $\delta_{N}$ quantifies the breaking of the mass degeneracy in the heavy-neutrino sector. Moreover, the Yukawa parameters $b$ and $c$ are not independent of $a$, but they obey the relations:

$$
b=a \sqrt{1+\frac{\delta_{N}}{m_{N}}}, \quad c=a \sqrt{1+\frac{2 \delta_{N}}{m_{N}}},
$$

such that the light neutrinos are exactly massless at the tree level, i.e., $\mathbf{m}_{\nu}=\mathbf{0}_{3}$, as can be easily determined from (1.1). Beyond the Born approximation, such a scenario leads to too large radiative neutrino masses in the $\nu_{R} \mathrm{SM}$ [31], in conflict with neutrino oscillation data, unless $\delta_{N} / m_{N} \lesssim 10^{-5}$, for $m_{N}=500 \mathrm{GeV}$ and light-toheavy neutrino mixing $\left(\mathbf{m}_{D}\right)_{11} / m_{N}=10^{-2}$. However, in the $\nu_{R} \mathrm{MSSM}$, we find that this constraint may be relaxed drastically by several orders of magnitude.

To showcase this relaxation of the constraint on the LNV parameter $\delta_{N}$, we consider a flavor scenario in which $\mathbf{A}_{\nu}$ has its own flavor structure that is independent of the neutrino Yukawa matrix $\mathbf{Y}_{\nu}$ as this can be inferred from (4.6). In this case, it is possible to find an appropriate form for $\mathbf{A}_{\nu}$ and a value for $b_{\nu}$, so as to reproduce the observed neutrino mass matrix $\mathbf{M}_{\nu_{L}}^{\text {exp }}$ given in (4.1). This is illustrated in Fig. 6, which shows the dependence of the average norm of $\mathbf{A}_{\nu}$, defined as

$$
\bar{A}_{\nu} \equiv \frac{1}{3} \operatorname{Tr}^{1 / 2}\left(\mathbf{A}_{\nu} \mathbf{A}_{\nu}^{\dagger}\right)
$$

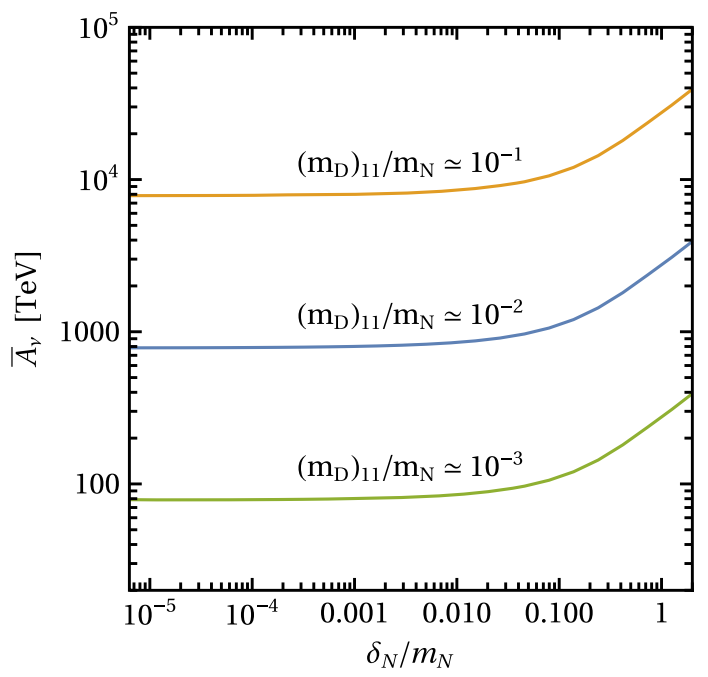

FIG. 6. The dependence of $\bar{A}_{\nu}$ [cf. (4.9)] on the LNV parameter $\delta_{N} / m_{N}$, for $a=0.5,0.05,0.005$ as shown from the upper to lower lines, respectively. The MSSM parameters are the same as in Table II (with $\tan \beta=2$ ), while the mass of the lightest neutrino is set to zero. on the LNV dimensionless parameter $\delta_{N} / m_{N}$, for different values of light-to-heavy neutrino mixing. Interestingly enough, it can be seen that large heavy neutrino mass differences $\delta_{N} / m_{N} \sim 10^{-1}$, with sizeable light-to-heavy neutrino mixing $\left(\mathbf{m}_{D}\right)_{11} / m_{N} \sim 10^{-2}$, are allowed, provided that the trilinear parameter $\bar{A}_{\nu}$ is sufficiently large, i.e., $\bar{A}_{\nu} \approx 1000 \mathrm{TeV}$.

In summary, our numerical estimates have revealed that larger values of LNV mass parameters, such as $\mu_{R}$ and $\delta_{N}$, are allowed in the $\nu_{R}$ MSSM than in the $\nu_{R} \mathrm{SM}$, while the squared light-to-heavy neutrino mixing as measured by $\lambda_{D}$ or $\left(\mathbf{m}_{D}\right)_{11} / m_{N}$ can be equally sizeable. Remarkably enough, this finding implies that signatures of LNV mediated by non-degenerate heavy Majorana neutrinos could be on the verge of being discovered with the upcoming LHC data [28].

\section{DISCUSSION}

We have studied supersymmetric scenarios of radiative neutrino masses that may occur in the minimal supersymmetric Standard Model with low-scale right-handed neutrinos $\left(\nu_{R} \mathrm{MSSM}\right)$. To have good control of the impact of the SUSY nonrenormalization theorems on the loopinduced neutrino masses, we have carefully performed all the Feynman-diagrammatic computations in the weak and flavor bases, rather in the mass basis. In this way, we have been able to identify a new mechanism for naturally suppressing the light neutrino masses beyond the traditional seesaw paradigm. In the context of the radiative inverse seesaw scenario first introduced and studied in [33], the smallness of the observed light neutrino masses may be the result of a soft SUSY-screening effect from a nearly supersymmetric singlet neutrino sector. An important consequence of this effect is that unlike in the non-supersymmetric scenario of [33], the singlet seesaw scale $m_{N}$ and the LNV scale $\mu_{R}$ can now be both comparable in size, e.g., of the electroweak order, while the size of the light-toheavy neutrino mixing squared, $\lambda_{D}$, can be enhanced up to the $10^{-2}$ level (cf. Fig. 4).

We note that the radiative generation of the light neutrino masses involves almost the full spectrum of particles of the $\nu_{R}$ MSSM. Specifically, the inclusion of third generation quarks and scalar quarks at the two-loop level are important to obtain an effective quartic Higgs coupling compatible with the SM Higgs-boson mass. If $R$-parity is conserved, the lightest right-handed sneutrino can be a successful dark matter candidate [76]. Hence, both the visible and DM sectors contribute, through one and higher loops, in order to generate the observed light neutrino masses. As such, the radiative screening mechanism presented in this paper is a consequence of pangenesis, as it requires almost the entire field content of the theory in order to be realized.

In this pangenic framework of the $\nu_{R}$ MSSM, the strict constraints [28,32] from light neutrino masses on LNV 
signatures from heavy Majorana neutrinos can be relaxed significantly, or even eliminated. On the other hand, possible observation of LNV signatures mediated by pairs of heavy Majorana neutrinos with masses $\sim\left(m_{N} \pm \mu_{R}\right)$ at high-energy colliders would give rise to renewed impetus in searches for supersymmetric right-handed sneutrinos in a very similar and highly correlated mass range.

Besides the above new aspects of collider phenomenology, minimal extensions of the $\nu_{R}$ MSSM not only can solve the infamous $\mu$-problem, but they can have profound cosmological implications as well, explaining the observed nearly scale-invariant cosmic microwave background spectrum and the flatness problem of the Universe through inflationary dynamics [77]. In such extensions, righthanded sneutrinos can become thermal DM particles [78]. Therefore, in light of the recent laboratory and cosmological data, it would be interesting to perform an updated, fully fledged analysis of such minimal extensions of the $\nu_{R}$ MSSM.

\section{ACKNOWLEDGMENTS}

The work of A. P. is supported in part by the LancasterManchester-Sheffield Consortium for Fundamental Physics, under STFC Research Grant No. ST/P000800/1. The work of P. C. dS. is funded by Agencia Nacional de Investigación y Desarrollo (ANID) through the Becas Chile Scholarship No. 72190359. The Feynman diagrams shown in this article were generated with the TikZ-Feynman package [79].

\section{APPENDIX A: THE HIGGS SECTOR OF THE $\nu_{R}$ MSSM AT TREE LEVEL}

Here we briefly review the SUSY limit in the $\nu_{R}$ MSSM, while describing our conventions for its Higgs sector. In fact, this sector becomes identical to the that of the MSSM at tree level. After SSB, the Higgs doublets $H_{u}$ and $H_{d}$ may be linearly expanded about their VEVs, $v_{u}$ and $v_{d}$, as follows:

$$
\begin{gathered}
H_{u}=\left(\begin{array}{c}
H_{u}^{+} \\
\frac{1}{\sqrt{2}}\left(v_{u}+\phi_{u}+i a_{u}\right)
\end{array}\right), \\
H_{d}=\left(\begin{array}{c}
\frac{1}{\sqrt{2}}\left(v_{d}+\phi_{d}+i a_{d}\right) \\
H_{d}^{-}
\end{array}\right) .
\end{gathered}
$$

In the MSSM, the electroweak symmetry breaking is in general connected to SUSY breaking. To see this, we start by analyzing the neutral part of the Higgs potential. This is given by

$$
\begin{aligned}
V_{H}^{0}= & \frac{1}{8}\left(g^{\prime 2}+g^{2}\right)\left(\left|H_{d}^{0}\right|^{2}-\left|H_{u}^{0}\right|^{2}\right)^{2}+\left(m_{H_{d}}^{2}+|\mu|^{2}\right)\left|H_{d}^{0}\right|^{2} \\
& +\left(m_{H_{u}}^{2}+|\mu|^{2}\right)\left|H_{u}^{0}\right|^{2}+\left(B \mu H_{d}^{0} H_{u}^{0}+\text { H.c. }\right),
\end{aligned}
$$

where $H_{u, d}^{0}$ are the neutral components of the Higgs doublets $H_{u, d}$. At its minimum, the scalar potential $V_{H}^{0}$ takes on the form

$$
\begin{aligned}
V_{H}^{\mathrm{vac}}= & \frac{1}{32}\left(g^{\prime 2}+g^{2}\right)\left(v_{d}^{2}-v_{u}^{2}\right)^{2}+\frac{1}{2}\left(m_{H_{d}}^{2}+|\mu|^{2}\right) v_{d}^{2} \\
& +\frac{1}{2}\left(m_{H_{u}}^{2}+|\mu|^{2}\right) v_{u}^{2}+\left(B \mu v_{d} v_{u}+\text { H.c. }\right) .
\end{aligned}
$$

Assuming that $B \mu$ is real, the minimization conditions for $V_{H}^{\mathrm{vac}}$ simplify to

$$
\begin{aligned}
& m_{H_{d}}^{2}+|\mu|^{2}=-B \mu \frac{v_{u}}{v_{d}}+\frac{1}{8}\left(g^{2}+g^{2}\right)\left(v_{u}^{2}-v_{d}^{2}\right), \\
& m_{H_{u}}^{2}+|\mu|^{2}=-B \mu \frac{v_{u}}{v_{d}}+\frac{1}{8}\left(g^{2}+g^{\prime 2}\right)\left(v_{d}^{2}-v_{u}^{2}\right) .
\end{aligned}
$$

It is now not difficult to see that when the soft SUSYbreaking terms are set to zero, these conditions can only be fulfilled for nonzero VEVs if $\mu=0$, which in turn implies that $v_{u}=v_{d}$. As a consequence, in the absence of any soft masses, the SUSY limit in the $\nu_{R}$ MSSM is attained for $\mu=0$ and $\tan \beta \equiv v_{u} / v_{d}=1$.

The part of the Lagrangian containing the $C P$-odd scalar masses is given by

$$
\mathcal{L}_{\mathrm{CP}-\mathrm{odd}}^{\text {mass }}=-\frac{1}{2}\left(\begin{array}{ll}
a_{u} & a_{d}
\end{array}\right)\left(\begin{array}{cc}
B \mu \cot \beta & B \mu \\
B \mu & B \mu \tan \beta
\end{array}\right)\left(\begin{array}{l}
a_{u} \\
a_{d}
\end{array}\right) .
$$

From this last expression, we see that one mass eigenstate is massless corresponding to the would-be Goldstone boson $G$ to be eaten by the longitudinal polarization of the $Z$ boson. Instead, the second mass eigenstate $A$ has a nonzero squared mass given by

$$
m_{A}^{2}=\frac{B \mu}{\sin \beta \cos \beta} .
$$

On the other hand, the $C P$-even mass Lagrangian reads

$$
\mathcal{L}_{\text {CP-even }}^{\text {mass }}=-\frac{1}{2}\left(\begin{array}{ll}
\phi_{u} & \phi_{d}
\end{array}\right)\left(\begin{array}{cc}
B \mu \cot \beta+\tilde{M}_{Z}^{2} \sin ^{2} \beta & -B \mu-M_{Z}^{2} \cos \beta \sin \beta \\
-B \mu-M_{Z}^{2} \cos \beta \sin \beta & B \mu \tan \beta+M_{Z}^{2} \cos ^{2} \beta
\end{array}\right)\left(\begin{array}{c}
\phi_{u} \\
\phi_{d}
\end{array}\right) .
$$


Note that in (A8), we promoted the up-type Higgs-boson quartic coupling from its tree-level value, $\lambda_{\text {tree }}=$ $\left(g^{2}+g^{\prime 2}\right) / 8$, to the effective coupling $\lambda_{\text {eff }}$ given in (3.5). Specifically, we have defined the mass parameter squared: $\tilde{M}_{Z}^{2} \equiv 2 \lambda_{\text {eff }}\left(v_{u}^{2}+v_{d}^{2}\right)$, which reduces to the standard treelevel result for $M_{Z}^{2}$, when $\lambda_{\text {eff }}$ is replaced with $\lambda_{\text {tree }}$. Denoting with $M_{\mathcal{S}}^{2}$ the $2 \times 2 C P$-even scalar mass matrix described by the Lagrangian in (A8), we may compute the two mass eigenstates, often called the light and heavy Higgs bosons, $h$ and $H$, as follows:

$$
m_{h, H}^{2}=\frac{1}{2}\left(\operatorname{Tr} M_{\mathcal{S}}^{2} \mp \sqrt{\left(\operatorname{Tr} M_{\mathcal{S}}^{2}\right)^{2}-4 \operatorname{det} M_{\mathcal{S}}^{2}}\right),
$$

with

$$
\begin{aligned}
\operatorname{Tr} M_{\mathcal{S}}^{2}= & m_{A}^{2}+\tilde{M}_{Z}^{2} \sin ^{2} \beta+M_{Z}^{2} \cos ^{2} \beta \\
\operatorname{det} M_{\mathcal{S}}^{2}= & m_{A}^{2}\left(M_{Z}^{2} \cos ^{4} \beta+\tilde{M}_{Z}^{2} \sin ^{4} \beta\right) \\
& -M_{Z}^{2}\left(2 m_{A}^{2}+M_{Z}^{2}-\tilde{M}_{Z}^{2}\right) \cos ^{2} \beta \sin ^{2} \beta
\end{aligned}
$$

In our analysis, we adopt a simplified approach, where the effective coupling $\lambda_{\text {eff }}$ is chosen such that the mass $m_{h}$ of the lightest $C P$-even scalar in the $\left(\nu_{R}\right) \mathrm{MSSM}$ coincides with the corresponding one for the observed SM-like Higgs resonance at the LHC [64], i.e., $m_{h}=125.38 \pm 0.14 \mathrm{GeV}$ [cf. Table II].

\section{APPENDIX B: NEUTRALINO AND HIGGS PROPAGATORS IN THE WEAK BASIS}

Here, we derive the analytic matrix structure of the neutralino propagator in the weak basis. To start with, we first quote the neutralino mass matrix in the basis $\left(\tilde{B}, \tilde{W}^{3}, \tilde{h}_{u}^{0}, \tilde{h}_{d}^{0}\right)^{\top}$, i.e.,

$M_{\chi^{0}}=\left(\begin{array}{cccc}M_{1} & 0 & \frac{1}{2} g^{\prime} v_{u} & -\frac{1}{2} g^{\prime} v_{d} \\ 0 & M_{2} & -\frac{1}{2} g v_{u} & \frac{1}{2} g v_{d} \\ \frac{1}{2} g^{\prime} v_{u} & -\frac{1}{2} g v_{u} & 0 & -\mu \\ -\frac{1}{2} g^{\prime} v_{d} & \frac{1}{2} g v_{d} & -\mu & 0\end{array}\right)$.

Then, in terms of $M_{\chi^{0}}$, we define the $4 \times 4$-dimensional matrix

$$
\mathcal{F}(k)=k^{2} \mathbf{1}_{4}-M_{\chi^{0}}^{*} M_{\chi^{0}} .
$$

We can prove that the left chiral component of the tree-level neutralino propagator can be written as

$$
P_{L} G_{\chi^{0}}^{(2)}(k) P_{L}=i M_{\chi^{0}} \mathcal{F}(k)^{-1} P_{L} .
$$

Employing the linear algebra relation,

$$
A^{-1}=\frac{1}{\operatorname{det} A} \operatorname{adj} A,
$$

which is valid for any invertible matrix $A$ with its adjunct denoted $\operatorname{as} \operatorname{adj} A$, we get

$$
\begin{aligned}
P_{L} G_{\chi^{0}}^{(2)}(k) P_{L} & =\frac{i}{\operatorname{det} \mathcal{F}} M_{\chi^{0}} \operatorname{adj} \mathcal{F} P_{L} \\
& =\frac{i}{\prod_{i=1}^{4}\left(k^{2}-m_{\chi_{i}^{0}}^{2}\right)} M_{\chi^{0}} \operatorname{adj} \mathcal{F} P_{L} .
\end{aligned}
$$

This expression proves very convenient when performing the Feynman parametrization of the loop integrals that involve this propagator.

Likewise, the $\mathrm{U}(1)_{Y}$-violating part of the $H_{u}$ propagator can be obtained after inverting the expression,

$$
\Gamma_{H}^{(2)}(p)=p^{2} \mathbf{1}_{4}-M_{H}^{2},
$$

and evaluating the respective entry, where $M_{H}^{2}$ is the Higgs mass matrix expressed in the weak basis, $\left(H_{d}^{0}, H_{u}^{0}, H_{d}^{0 *}, H_{u}^{0 *}\right)^{\top}$. In this way, we may derive

$G_{H_{u}^{0 *} H_{u}^{0 *}}^{(2)}(p)=\frac{i \tilde{M}_{Z}^{2} \sin ^{2} \beta\left(p^{2}+m_{A}^{2} \cos 2 \beta\right)^{2}}{2 p^{2}\left(p^{2}-m_{A}^{2}\right)\left(p^{2}-m_{h}^{2}\right)\left(p^{2}-m_{H}^{2}\right)}$.

In this last expression, the $Z$-boson mass squared, $M_{Z}^{2}$, has been replaced with an effective mass parameter $\tilde{M}_{Z}^{2}$. This enables us to take into account the quantum corrections to the quartic coupling $\left(H_{u}^{\dagger} H_{u}\right)^{2}$, in agreement with our simplified approach discussed in Appendix A.

\section{APPENDIX C: LOOP CORRECTION COEFFICIENTS}

Here we list key auxiliary expressions that we have used in Sec. III. For convenience, we define

$$
\begin{aligned}
\frac{1}{v_{2}} & {\left[g\left(M_{\chi^{0}} \operatorname{adj} \mathcal{F}\right)_{\tilde{h}_{u}^{0} \tilde{W}^{3}}-g^{\prime}\left(M_{\chi^{0}} \operatorname{adj} \mathcal{F}\right)_{\tilde{h}_{u}^{0} \tilde{B}}\right] } \\
& =A k^{6}+B k^{4}+C k^{2}+D
\end{aligned}
$$

where $\mathcal{F}$ is a $4 \times 4$ matrix given in (B2) and the coefficients $A, B, C, D$ are found to be

$$
\begin{aligned}
A= & -\frac{1}{2}\left(g^{\prime 2}+g^{2}\right), \\
B= & \frac{1}{2} M_{Z}^{2}+\frac{g^{\prime 2}}{2}\left(|\mu|^{2}+\left|M_{2}\right|^{2}-\mu M_{1} \cot \beta\right) \\
& +\frac{g^{2}}{2}\left(|\mu|^{2}+\left|M_{1}\right|^{2}-\mu M_{2} \cot \beta\right),
\end{aligned}
$$




$$
\begin{aligned}
C= & \frac{|\mu|^{2}}{2}\left[\mu \cot \beta\left(g^{\prime 2} M_{1}+g^{2} M_{2}\right)-g^{\prime 2}\left|M_{2}\right|^{2}-g^{2}\left|M_{1}\right|^{2}-2 v_{d}^{2}\left(g^{\prime 2}+g^{2}\right)\right] \\
& -\left(g^{\prime 2} M_{2}^{*}+g^{2} M_{1}^{*}\right)\left[\left(v_{d}^{2}+v_{u}^{2}\right)\left(g^{2} M_{1}+g^{\prime 2} M_{2}\right)-4 \mu M_{1} M_{2} \cot \beta\right], \\
D= & \frac{|\mu|^{2}}{4}\left(g^{\prime 2} M_{2}^{*}-g^{2} M_{1}^{*}\right)\left[\left(g^{\prime 2} M_{2}+g^{2} M_{1}\right) v_{d} v_{u}-2 \mu M_{1} M_{2}\right] \cot \beta .
\end{aligned}
$$

Similarly, we may define

$$
\frac{1}{2}\left[g^{\prime 2}\left(M_{\chi^{0}} \operatorname{adj} \mathcal{F}\right)_{\tilde{B} \tilde{B}}+g^{2}\left(M_{\chi^{0}} \operatorname{adj} \mathcal{F}\right)_{\tilde{W}^{3} \tilde{W}^{3}}\right]=A^{\prime} k^{6}+B^{\prime} k^{4}+C^{\prime} k^{2}+D^{\prime},
$$

where

$$
\begin{aligned}
A^{\prime}= & \frac{1}{2}\left(g^{\prime 2} M_{1}+g^{2} M_{2}\right), \\
B^{\prime}= & -\frac{g^{\prime 2}}{2} M_{1}\left|M_{2}\right|^{2}-\frac{g^{2}}{2} M_{2}\left|M_{1}\right|^{2}-|\mu|^{2}\left(g^{\prime 2} M_{1}+g^{2} M_{2}\right)-\frac{g^{\prime 2} g^{2}}{4}\left(M_{1}+M_{2}\right)\left(v_{d}^{2}+v_{u}^{2}\right) \\
& +\frac{\mu^{*}}{4}\left(g^{\prime 4}+g^{4}\right) v_{d} v_{u}, \\
C^{\prime}= & \frac{|\mu|^{4}}{2}\left(g^{\prime 2} M_{1}+g^{2} M_{2}\right)-\frac{\mu^{*}}{4}|\mu|^{2}\left(g^{\prime 4}+g^{4}\right) v_{d} v_{u}-\frac{g^{\prime 2}}{4}\left|M_{2}\right|^{2} \mu^{*}\left(g^{\prime 2} v_{d} v_{u}-4 \mu M_{1}\right) \\
& -\frac{g^{2}}{4}\left|M_{1}\right|^{2} \mu^{*}\left(g^{2} v_{d} v_{u}-4 \mu M_{1}\right)+\frac{|\mu|^{2}}{4} g^{\prime 2} g^{2}\left(M_{1}+M_{2}\right)\left(v_{d}^{2}+v_{u}^{2}\right) \\
& -\frac{\mu^{*}}{4} g^{\prime 2} g^{2}\left(M_{1} M_{2}^{*}+M_{1}^{*} M_{2}\right) v_{d} v_{u}+\frac{g^{\prime 2} g^{2}}{16}\left(g^{2} M_{1}+g^{\prime 2} M_{2}\right)\left(v_{d}^{2}+v_{u}^{2}\right)^{2} \\
& -\frac{\mu}{2} g^{2} g^{\prime 2} M_{1} M_{2} v_{d} v_{u}, \\
D^{\prime}= & -\frac{|\mu|^{2}}{4}\left[\mu^{*}\left(g^{\prime 2} M_{2}+g^{2} M_{1}\right)-g^{\prime 2} g^{2} v_{d} v_{u}\right]\left[\left(g^{\prime 2} M_{2}+g^{2} M_{1}\right) v_{d} v_{u}-2 \mu M_{1} M_{2}\right] .
\end{aligned}
$$

Finally, we have used the expression

$$
\frac{2}{v_{u}^{2}}\left[g^{2}\left(M_{\chi^{0}} \operatorname{adj} \mathcal{F}\right)_{\tilde{h}_{u} \tilde{h}_{u}}\right]=A^{\prime \prime} k^{6}+B^{\prime \prime} k^{4}+C^{\prime \prime} k^{2}+D^{\prime \prime},
$$

where

$$
\begin{aligned}
A^{\prime \prime}= & 0, \\
B^{\prime \prime}= & 4\left(g^{\prime 2} M_{1}^{*}+g^{2} M_{2}^{*}\right)+8 \mu \cot \beta\left(g^{\prime 2}+g^{2}\right), \\
C^{\prime \prime}= & 2 g_{1}^{4} \mu\left|M_{2}\right|^{2} v_{d}^{2} \cot \beta+2 \mu g_{1}^{2} M_{1} M_{2}^{*} \cot ^{2} \beta\left(g_{2}^{2} v_{d} v_{u}-2 \mu M_{2}\right) \\
& +2 g_{2}^{4} \mu\left|M_{1}\right|^{2} v_{d}^{2} \cot \beta+2 \mu g_{2}^{2} M_{2} M_{1}^{*} \cot ^{2} \beta\left(g_{1}^{2} v_{d} v_{u}-2 \mu M_{1}\right), \\
D^{\prime \prime}= & -8 \mu v_{d} v_{u}\left(g^{\prime 2}\left|M_{2}\right|^{2}+g^{2}\left|M_{1}\right|^{2}\right)-4 v_{u}^{2} M_{1}^{*} M_{2}^{*}\left(g^{\prime 2} M_{2}+g^{2} M_{1}\right) \\
& +4 \mu^{2} v_{d}^{2}\left(g^{\prime 2} M_{1}+g^{2} M_{2}\right)-2\left(g^{\prime 2}+g^{2}\right)^{2} \mu v_{d}^{3} v_{u} .
\end{aligned}
$$


[1] D. V. Volkov and V. P. Akulov, Is the neutrino a Goldstone particle?, Phys. Lett. 46B, 109 (1973).

[2] J. Wess and B. Zumino, Supergauge transformations in fourdimensions, Nucl. Phys. B70, 39 (1974).

[3] H. P. Nilles, Supersymmetry, supergravity and particle physics, Phys. Rep. 110, 1 (1984).

[4] M. T. Grisaru, W. Siegel, and M. Rocek, Improved methods for supergraphs, Nucl. Phys. B159, 429 (1979).

[5] J. Wess and J. Bagger, Supersymmetry and Supergravity (Princeton University Press, Princeton, NJ, USA, 1992).

[6] A. Djouadi, The Anatomy of electro-weak symmetry breaking. II. The Higgs bosons in the minimal supersymmetric model, Phys. Rep. 459, 1 (2008).

[7] S. Dimopoulos and H. Georgi, Softly broken supersymmetry and SU(5), Nucl. Phys. B193, 150 (1981).

[8] H. E. Haber and G. L. Kane, The search for supersymmetry: Probing physics beyond the standard model, Phys. Rep. 117, 75 (1985).

[9] S. Bertolini, F. Borzumati, A. Masiero, and G. Ridolfi, Effects of supergravity induced electroweak breaking on rare $B$ decays and mixings, Nucl. Phys. B353, 591 (1991).

[10] F. Deppisch and J. W. F. Valle, Enhanced lepton flavor violation in the supersymmetric inverse seesaw model, Phys. Rev. D 72, 036001 (2005).

[11] A. Ilakovac and A. Pilaftsis, Supersymmetric lepton flavor violation in low-scale seesaw models, Phys. Rev. D 80, 091902 (2009).

[12] A. Ilakovac, A. Pilaftsis, and L. Popov, Charged lepton flavor violation in supersymmetric low-scale seesaw models, Phys. Rev. D 87, 053014 (2013).

[13] M. Carena, G. F. Giudice, and C. E. M. Wagner, Constraints on supersymmetric models from the muon anomalous magnetic moment, Phys. Lett. B 390, 234 (1997).

[14] A. Ilakovac, A. Pilaftsis, and L. Popov, Lepton dipole moments in supersymmetric low-scale seesaw models, Phys. Rev. D 89, 015001 (2014).

[15] Y. Fukuda et al., Evidence for Oscillation of Atmospheric Neutrinos, Phys. Rev. Lett. 81, 1562 (1998).

[16] Q. R. Ahmad et al., Measurement of the Rate of $\nu_{e}+d \rightarrow$ $p+p+e^{-}$Interactions Produced by ${ }^{8} B$ Solar Neutrinos at the Sudbury Neutrino Observatory, Phys. Rev. Lett. 87, 071301 (2001).

[17] Q. R. Ahmad et al., Direct Evidence for Neutrino Flavor Transformation from Neutral Current Interactions in the Sudbury Neutrino Observatory, Phys. Rev. Lett. 89, 011301 (2002).

[18] P. Minkowski, $\mu \rightarrow e \gamma$ at a rate of one out of $10^{9}$ muon decays? Phys. Lett. 67B, 421 (1977).

[19] T. Yanagida, Horizontal gauge symmetry and masses of neutrinos, Conf. Proc. C 7902131, 95 (1979).

[20] M. Gell-Mann, P. Ramond, and R. Slansky, Complex spinors and unified theories, Conf. Proc. C 790927, 315 (1979), https://inspirehep.net/literature/9686.

[21] R. N. Mohapatra and G. Senjanovic, Neutrino Mass and Spontaneous Parity Nonconservation, Phys. Rev. Lett. 44, 912 (1980).

[22] R. N. Mohapatra and G. Senjanovic, Neutrino masses and mixings in gauge models with spontaneous parity violation, Phys. Rev. D 23, 165 (1981).
[23] J. Schechter and J. W. F. Valle, Neutrino masses in $\mathrm{SU}(2) \times \mathrm{U}(1)$ theories, Phys. Rev. D 22, 2227 (1980).

[24] G. Lazarides, Q. Shafi, and C. Wetterich, Proton lifetime and fermion masses in an SO(10) model, Nucl. Phys. B181, 287 (1981).

[25] M. Magg and C. Wetterich, Neutrino mass problem and gauge hierarchy, Phys. Lett. 94B, 61 (1980).

[26] R. Foot, H. Lew, X. G. He, and G. C. Joshi, See-saw neutrino masses induced by a triplet of leptons, Z. Phys. C 44, 441 (1989).

[27] E. Majorana, Teoria simmetrica dell'elettrone e del positrone, Nuovo Cimento 14, 171 (1937).

[28] F. F. Deppisch, P. S. Bhupal Dev, and A. Pilaftsis, Neutrinos and collider physics, New J. Phys. 17, 075019 (2015).

[29] R. N. Mohapatra, Mechanism for Understanding Small Neutrino Mass in Superstring Theories, Phys. Rev. Lett. 56, 561 (1986).

[30] R. N. Mohapatra and J. W. F. Valle, Neutrino mass and baryon number nonconservation in superstring models, Phys. Rev. D 34, 1642 (1986).

[31] A. Pilaftsis, Radiatively induced neutrino masses and large Higgs neutrino couplings in the standard model with Majorana fields, Z. Phys. C 55, 275 (1992).

[32] J. Kersten and A. Y. Smirnov, Right-handed neutrinos at CERN LHC and the mechanism of neutrino mass generation, Phys. Rev. D 76, 073005 (2007).

[33] P.S. Bhupal Dev and A. Pilaftsis, Minimal radiative neutrino mass mechanism for inverse seesaw models, Phys. Rev. D 86, 113001 (2012).

[34] W. Grimus and M. Loschner, Renormalization of the multiHiggs-doublet Standard Model and one-loop lepton mass corrections, J. High Energy Phys. 11 (2018) 087.

[35] Y. Cai, J. Herrero-Garca, M. A. Schmidt, A. Vicente, and R. R. Volkas, From the trees to the forest: A review of radiative neutrino mass models, Front. Phys. 5, 63 (2017).

[36] A. Datta, M. Guchait, and A. Pilaftsis, Probing lepton number violation via Majorana neutrinos at hadron supercolliders, Phys. Rev. D 50, 3195 (1994).

[37] S. Bray, J. S. Lee, and A. Pilaftsis, Resonant $C P$ violation due to heavy neutrinos at the LHC, Nucl. Phys. B786, 95 (2007).

[38] A. Atre, T. Han, S. Pascoli, and B. Zhang, The search for heavy Majorana neutrinos, J. High Energy Phys. 05 (2009) 030 .

[39] G. Cvetic, C. Dib, S. K. Kang, and C. S. Kim, Probing Majorana neutrinos in rare $K$ and $D, D_{s}, \mathrm{~B}, B_{c}$ meson decays, Phys. Rev. D 82, 053010 (2010).

[40] P. S. Bhupal Dev, A. Pilaftsis, and U.-K. Yang, New Production Mechanism for Heavy Neutrinos at the LHC, Phys. Rev. Lett. 112, 081801 (2014).

[41] A. Das, P. Konar, and A. Thalapillil, Jet substructure shedding light on heavy Majorana neutrinos at the LHC, J. High Energy Phys. 02 (2018) 083.

[42] A. Bhardwaj, A. Das, P. Konar, and A. Thalapillil, Looking for minimal inverse seesaw scenarios at the LHC with jet substructure techniques, J. Phys. G 47, 075002 (2020).

[43] A. Dedes, H. E. Haber, and J. Rosiek, Seesaw mechanism in the sneutrino sector and its consequences, J. High Energy Phys. 11 (2007) 059. 
[44] M. Hirsch, T. Kernreiter, J. C. Romao, and A. Villanova del Moral, Minimal supersymmetric inverse seesaw: Neutrino masses, lepton flavour violation and LHC phenomenology, J. High Energy Phys. 01 (2010) 103.

[45] W. G. Hollik, Neutrinos meet supersymmetry: Quantum aspects of neutrinophysics in supersymmetric theories, Ph.D. thesis, KIT, Karlsruhe, Dept. Phys., 2015.

[46] J. Hisano, T. Moroi, K. Tobe, M. Yamaguchi, and T. Yanagida, Lepton flavor violation in the supersymmetric standard model with seesaw induced neutrino masses, Phys. Lett. B 357, 579 (1995).

[47] Y. Grossman and H. E. Haber, Sneutrino Mixing Phenomena, Phys. Rev. Lett. 78, 3438 (1997).

[48] J. A. Casas and A. Ibarra, Oscillating neutrinos and $\mu \rightarrow e, \gamma$, Nucl. Phys. B618, 171 (2001).

[49] Y. Farzan, Effects of the neutrino B term on slepton mixing and electric dipole moments, Phys. Rev. D 69, 073009 (2004).

[50] E. J. Chun, A. Masiero, A. Rossi, and S. K. Vempati, A predictive seesaw scenario for EDMs, Phys. Lett. B 622, 112 (2005).

[51] D. A. Demir and Y. Farzan, Can measurements of electric dipole moments determine the seesaw parameters?, J. High Energy Phys. 10 (2005) 068.

[52] E. Arganda and M. J. Herrero, Testing supersymmetry with lepton flavor violating tau and mu decays, Phys. Rev. D 73, 055003 (2006).

[53] S. Heinemeyer, M. J. Herrero, S. Penaranda, and A. M. Rodriguez-Sanchez, Higgs boson masses in the MSSM with heavy Majorana neutrinos, J. High Energy Phys. 05 (2011) 063.

[54] A. Salam and J. Strathdee, Supersymmetry and superfields, Fortschr. Phys. 26, 57 (1978).

[55] S. Banerjee, P. S. Bhupal Dev, S. Mondal, B. Mukhopadhyaya, and S. Roy, Invisible Higgs decay in a supersymmetric inverse seesaw model with light sneutrino dark matter, J. High Energy Phys. 10 (2013) 221.

[56] H. An, P. S. Bhupal Dev, Y. Cai, and R. N. Mohapatra, Sneutrino Dark Matter in Gauged Inverse Seesaw Models for Neutrinos, Phys. Rev. Lett. 108, 081806 (2012).

[57] J. Guo, Z. Kang, T. Li, and Y. Liu, Higgs boson mass and complex sneutrino dark matter in the supersymmetric inverse seesaw models, J. High Energy Phys. 02 (2014) 080.

[58] E. Arganda, M. J. Herrero, X. Marcano, and C. Weiland, Imprints of massive inverse seesaw model neutrinos in lepton flavor violating Higgs boson decays, Phys. Rev. D 91, 015001 (2015).

[59] B. A. Kniehl and A. Pilaftsis, Mixing renormalization in Majorana neutrino theories, Nucl. Phys. B474, 286 (1996).

[60] A. Pilaftsis, Gauge and scheme dependence of mixing matrix renormalization, Phys. Rev. D 65, 115013 (2002).

[61] J. R. Ellis, G. Ridolfi, and F. Zwirner, Radiative corrections to the masses of supersymmetric Higgs bosons, Phys. Lett. B 257, 83 (1991).
[62] H. E. Haber and R. Hempfling, Can the Mass of the Lightest Higgs Boson of the Minimal Supersymmetric Model be Larger than $\mathrm{m}(\mathrm{Z})$ ?, Phys. Rev. Lett. 66, 1815 (1991).

[63] Y. Okada, M. Yamaguchi, and T. Yanagida, Upper bound of the lightest Higgs boson mass in the minimal supersymmetric standard model, Prog. Theor. Phys. 85, 1 (1991).

[64] A. M. Sirunyan et al., A measurement of the Higgs boson mass in the diphoton decay channel, Phys. Lett. B 805, 135425 (2020).

[65] A. Pilaftsis and C.E. M. Wagner, Higgs bosons in the minimal supersymmetric standard model with explicit $C P$ violation, Nucl. Phys. B553, 3 (1999).

[66] J. S. Lee, M. Carena, J. Ellis, A. Pilaftsis, and C. E. M. Wagner, CPsuperH2.0: An improved computational tool for Higgs phenomenology in the MSSM with explicit $C P$ violation, Comput. Phys. Commun. 180, 312 (2009).

[67] S. Heinemeyer, W. Hollik, H. Rzehak, and G. Weiglein, The Higgs sector of the complex MSSM at two-loop order: QCD contributions, Phys. Lett. B 652, 300 (2007).

[68] M. Carena, J. Ellis, J. S. Lee, A. Pilaftsis, and C. E. M. Wagner, $C P$ violation in heavy MSSM Higgs scenarios, J. High Energy Phys. 02 (2016) 123.

[69] G. Passarino and M. J. G. Veltman, One loop corrections for e+ e- annihilation into mu+ mu- in the Weinberg model, Nucl. Phys. B160, 151 (1979).

[70] B. Pontecorvo, Inverse beta processes and nonconservation of lepton charge, Sov. Phys. JETP 7, 172 (1958), http:// www.jetp.ac.ru/cgi-bin/e/index/e/7/1/p172?a=list.

[71] Z. Maki, M. Nakagawa, and S. Sakata, Remarks on the unified model of elementary particles, Prog. Theor. Phys. 28, 870 (1962).

[72] P. de Salas, D. Forero, S. Gariazzo, P. Martínez-Miravé, O. Mena, C. Ternes, M. Tórtola, and J. Valle, 2020 Global reassessment of the neutrino oscillation picture, arXiv:2006 .11237.

[73] J. D. Wells, Implications of supersymmetry breaking with a little hierarchy between gauginos and scalars, arXiv:hep-ph/ 0306127.

[74] N. Arkani-Hamed and S. Dimopoulos, Supersymmetric unification without low energy supersymmetry and signatures for fine-tuning at the LHC, J. High Energy Phys. 06 (2005) 073.

[75] G. F. Giudice and A. Romanino, Split supersymmetry, Nucl. Phys. B699, 65 (2004); Erratum, B706, 487 (2005).

[76] S. Gopalakrishna, A. de Gouvea, and W. Porod, Righthanded sneutrinos as nonthermal dark matter, J. Cosmol. Astropart. Phys. 05 (2006) 005.

[77] B. Garbrecht, C. Pallis, and A. Pilaftsis, Anatomy of F(D)term hybrid inflation, J. High Energy Phys. 12 (2006) 038.

[78] F. Deppisch and A. Pilaftsis, Thermal right-handed sneutrino dark matter in the $\mathrm{F}(\mathrm{D})$-term model of hybrid inflation, J. High Energy Phys. 10 (2008) 080.

[79] J. Ellis, TikZ-Feynman: Feynman diagrams with TikZ, Comput. Phys. Commun. 210, 103 (2017). 